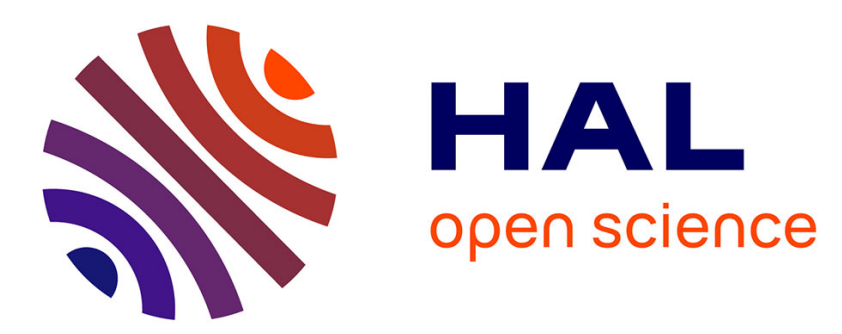

\title{
Minimal Time Control of the Two Tanks Gradostat Model Under a Cascade Input Constraint
}

Térence Bayen, Alain Rapaport, Matthieu Sebbah

\section{To cite this version:}

Térence Bayen, Alain Rapaport, Matthieu Sebbah. Minimal Time Control of the Two Tanks Gradostat Model Under a Cascade Input Constraint. SIAM Journal on Control and Optimization, 2014, 52 (4), pp.2568-2594. 10.1137/130950379 . hal-00798651v3

\section{HAL Id: hal-00798651 https://hal.science/hal-00798651v3}

Submitted on 2 Jun 2014

HAL is a multi-disciplinary open access archive for the deposit and dissemination of scientific research documents, whether they are published or not. The documents may come from teaching and research institutions in France or abroad, or from public or private research centers.
L'archive ouverte pluridisciplinaire HAL, est destinée au dépôt et à la diffusion de documents scientifiques de niveau recherche, publiés ou non, émanant des établissements d'enseignement et de recherche français ou étrangers, des laboratoires publics ou privés. 


\title{
MINIMAL TIME CONTROL OF THE TWO TANKS GRADOSTAT MODEL UNDER A CASCADE INPUT CONSTRAINT
}

\author{
T. BAYEN ${ }^{\dagger}$, A. RAPAPORT ${ }^{\ddagger}$, AND M. SEBBAH ${ }^{\S} \|$
}

\begin{abstract}
We study the minimum time control problem of a series of two interconnected chemostats under the input constraint $u_{2} \leq u_{1}$, where $u_{i}$ are the respective dilution rates in the tanks. This constraint brings controllability issues in the study of the optimal strategies. We overcome this difficulty by splitting the state domain into two sub-domains, one with no lack of controllability of the target, and its complement where any optimal trajectory satisfies $u_{1}=u_{2}$. We explicitly compute the complete optimal synthesis that depends on the position of the target with respect to a semi-permeable curve that passes through a steady-state singular point.
\end{abstract}

Key words. optimal control, minimal time problem, Pontryagin's Maximum Principle, optimal synthesis, chemostat model, gradostat model, non-linear controllability, semi-permeability.

AMS subject classifications. 49J15, 49K15, 49N25.

1. Introduction. The concept of the chemostat has been simultaneously introduced by Novick \& Szilard and Monod in the fifties, to describe continuous culture of micro-organisms. The associated mathematical model is expressed by the following system of two differential equations (see for instance [34]):

$$
\left\{\begin{array}{l}
\dot{b}=(\mu(s)-u) b \\
\dot{s}=-\mu(s) b+u\left(s_{i n}-s\right)
\end{array}\right.
$$

where $b$ and $s$ for the biomass and nutrient concentrations, respectively. The function $\mu(\cdot)$ is the specific growth rate of the micro-organisms (most often a monotonic increasing function). The inlet concentration of nutrient $s_{i n}$ and the dilution rate $u$ are the system inputs. Note that in the above equations the yield coefficient of the bio-conversion is not explicitly given, because it has been chosen to be equal to one (that can be always done without any loss of generality).

The chemostat is typically designed to be operated at steady state, that is for state $\left(b_{e q}, s_{e q}\right)$ such that $\mu\left(s_{e q}\right)=u$ and $b_{e q}=s_{i n}-s_{e q}$. Such a model is often considered as a good representation of the operation of bioreactors in biotechnology or wastewater industries [16], or for ecological investigations [36] of the growth of micro-organisms in natural environments, such as mountain lakes.

Cascades of chemostats or bioreactors are largely considered in microbiology ("gradostats" [17, 26, 33, 35]) or in bio-processes ("serial tanks" [2]), because of their ability to create a gradient of resources (see also $[13,19]$ ). Such gradients are expected to be more realistic to mimic real environment for studying micro-organisms growth $[10,22]$. In biotechnology, series of bioreactors are also known to be more efficient for

\footnotetext{
${ }^{\dagger}$ Université Montpellier 2, CC 051, 34095 Montpellier cedex 5, France (tbayen@math.univmontp2.fr).

${ }^{\ddagger}$ UMR INRA-SupAgro 729 'MISTEA', 2 place Viala 34060 gou]Montpellier, France (rapaport@supagro.inra.fr).

§Universidad Tecnica Federico Santa Maria, Departamento de Matematica, Avenida Espana 1680, Valparaiso, Chile (matthieu.sebbah@usm.cl).

`INRA-Inria 'MODEMIC' team, Inria Sophia-Antipolis Méditerranée, route des Lucioles, 06902 Sophia-Antipolis, France.

"Bionature team, Communication and Information Research and Innovation Center (CIRIC), Inria Chile, Avenida Apoquindo 2827, Las Condes, Santiago, Chile.
} 
the resource conversion at steady state than single tanks $[2,21,23,30]$. The extension of the chemostat model to a cascade of two chemostats is straightforwardly given by the following set of differential equations

$$
\left\{\begin{array}{l}
\dot{b}_{1}=\left(\mu\left(s_{1}\right)-u_{1}\right) b_{1} \\
\dot{s}_{1}=-\mu\left(s_{1}\right) b_{1}+u_{1}\left(s_{i n}-s_{1}\right) \\
\dot{b}_{2}=\left(\mu\left(s_{2}\right)-u_{2}\right) b_{2}+u_{2} b_{1} \\
\dot{s}_{2}=-\mu\left(s_{2}\right) b_{2}+u_{2}\left(s_{1}-s_{2}\right)
\end{array}\right.
$$

Note that the input variables $u_{1}, u_{2}$ are not independent, because the inlet flow of the second tank cannot be larger than the output flow of the first tank. When the volumes of the tanks are equal (what we shall assume without loss of generality), this amounts to consider control inputs $u$ that satisfy the input cascade constraint:

$$
u \in U:=\left\{\left(u_{1}, u_{2}\right) \in \mathbb{R}^{2} \mid 0 \leq u_{2} \leq u_{1} \leq u_{\max }\right\}
$$

As for the chemostat, the gradostat device is typically designed to be operated at steady state. Typical practical issues raised in laboratory or in industrial context environment are related to the changes of the operation point from one current steady state to another. This problem has been for instance raised in recent investigations on wine fermentation processes [11] during the transient operations, where each tank corresponds to a precise metabolic state of the micro-organisms related to the level of substrate concentration, that one would like to adjust in each tank. Typically, a steady state corresponds to a particular physiological state of the bacteria, and practitioners would like to switch from state to another one.

It is worth noting from equations (1.1) that any steady state belongs to the hyperplane $s_{i}+b_{i}=s_{i n}(i=1,2)$, which is invariant for any control input $u(\cdot)$. Note also that these hyperplanes are attractive for any constant non-null control inputs [34]. For our study the dynamics (1.1) can then be reduced to the following planar one:

$$
\left\{\begin{array}{l}
\dot{s}_{1}=-\nu\left(s_{1}\right)+u_{1}\left(s_{i n}-s_{1}\right), \\
\dot{s}_{2}=-\nu\left(s_{2}\right)+u_{2}\left(s_{1}-s_{2}\right)
\end{array}\right.
$$

where we have set $\nu(s):=\mu(s)\left(s_{i n}-s\right)$ and $b_{i}=s_{i n}-s_{i}$. In another context, the same dynamics can equivalently be rewritten in $x_{i}$ variables (formerly $x_{i}:=s_{i n}-s_{i}$ ):

$$
\left\{\begin{array}{l}
\dot{x}_{1}=g\left(x_{1}\right)-u_{1} x_{1} \\
\dot{x}_{2}=g\left(x_{2}\right)+u_{2}\left(x_{1}-x_{2}\right)
\end{array}\right.
$$

where we have set $g(x):=\mu\left(s_{\text {in }}-x\right) x$. Such a model could also represent stocks of natural resources such as fisheries, where a typical instance of the growth function $g(\cdot)$ is the logistic law [9]. Here $x_{i}$ and $u_{i}(i=1,2)$ hold for the population densities in two areas and the corresponding harvesting efforts, respectively. The total harvesting is then $h=\left(u_{1}-u_{2}\right) x_{1}+u_{2} x_{2}$, where a part of the harvest $u_{2} x_{1}$ in the first area is reintroduced in the second one, similarly to mathematical models of the management of marine protected areas $[1,14,20,32]$.

The problem of the change of the operation point then amounts to choose a twodimensional control input $u(\cdot)=\left(u_{1}(\cdot), u_{2}(\cdot)\right)$ that drives the system (1.3) towards a new target $\bar{s}=\left(\bar{s}_{1}, \bar{s}_{2}\right)$. It is important to mention that both control inputs are 
necessary in order to drive the system to a given equilibrium for $s_{1}$ and $s_{2}$. For this objective, one can simply adjust the control input $u$ to the value that corresponds to the new desired steady state, and wait for the asymptotic convergence of the state. Instead, a feedback strategy for the minimal time criterion appears to be a natural choice for the practitioners, since it allows to gain time while manipulating the variable $u$ (as this is often considered for other problems in the same application fields $[12,18,29])$. Our objective in this paper is therefore to find a feedback control $s \mapsto u[s]$ steering (1.3) from any initial state to a given target $\bar{s}$ in minimal time.

For minimal time control problems in the plane with control affine dynamics, the theory has been mostly developed for control sets in $\ell_{1}$ or $\ell_{2}$-balls (see e.g. [5, 6, 25]). Note that the input constraint (1.2) has a different geometry, as it is is not diffeomorphic to any $\ell_{1}$ or $\ell_{2}$-balls and has "corners". This unusual feature leads to new local controllability issues for the synthesis of optimal feedbacks, that we indeed investigate in the present work.

One can easily check that there exists a (forwardly) invariant domain $\mathcal{D}$ for (1.3), and we shall restrict our study for initial conditions in this domain. Given a target point $\bar{s}$ in $\mathcal{D}$, our main key is to characterize a subset $\mathcal{C}(\bar{s}) \subset \mathcal{D}$ that fulfills the following properties:

- $\bar{s} \in \mathcal{C}(\bar{s})$ is reachable from any initial condition in $\mathcal{C}(\bar{s})$, and any optimal trajectory stays in $\mathcal{C}(\bar{s})$,

- any optimal control input from an initial condition outside $\mathcal{C}(\bar{s})$ is such that $u_{1}(t)=u_{2}(t)$ almost everywhere.

This feature allows to decompose the original optimal feedback synthesis problem in $\mathcal{D}$ into two simpler sub-problems:

1. the minimal time control problem in $\mathcal{C}(\bar{s})$ with two constrained control inputs, without any controllability issue,

2. the minimal time control problem in $\mathcal{D}$ with $\mathcal{C}(\bar{s})$ as a target, with a scalar control input, but with some controllability issues.

The paper is organized as follows. In the first section, we introduce hypotheses and apply Pontryagin's Maximum Principle (PMP). We also introduce the set $\mathcal{C}(\bar{s})$, and provide some qualitative properties of its boundary. In the second section, we give the optimal synthesis for initial conditions in the set $\mathcal{C}(\bar{s})$ (Proposition 3.3). We show that there exist infinitely many optimal trajectories steering a point in this set to the target. In the third section, we show using the PMP that for initial conditions outside the set $\mathcal{C}(\bar{s})$, the optimal control necessarily fulfills $u_{1}=u_{2}$. This reduction allows to use properties of affine systems in the plane with one input in order to obtain the optimal synthesis (see e.g. [6]). Our analysis is thus devoted to the case of a cascade of two tanks only. We show that either the target is never reached from the set $\mathcal{D} \backslash \mathcal{C}(\bar{s})$ (case I), or it can be reached from any initial condition outside $\mathcal{C}(\bar{s})$, and the optimal strategy is singular (case II). In this section, we exhibit a partition of the domain $\mathcal{D}$ into two sub-domains $A$ and $B$. We show that if the target is in $A$ (resp. in $B$ ) then, the optimal synthesis is as in case I (resp. case II). This decomposition of $\mathcal{D}$ is based on properties of semi-permeable curves $([7,8,31])$ and steady-state singular points that are the intersection points between the non-controllability set and the singular locus [6]. Finally Theorem 4.17 is our main result and provides a complete optimal synthesis of the problem. The last section is devoted to numerical simulations for particular choices of the growth function $\mu$. 
2. Preliminaries. In this section, we introduce the set $\mathcal{C}(\bar{s})$ for a given target $\bar{s}$, and we give some properties of this set. We also apply the Pontryagin's Maximum Principle on the minimum time problem.

2.1. Hypotheses and notations. We assume the usual hypotheses on the function $\mu(\cdot)$ :

Hypothesis H1. $\mu(\cdot)$ is a bounded $C^{2}$ non-negative increasing function defined $[0,+\infty)$ with $\mu(0)=0$.

A typical example is when $\mu$ is of Monod type, see [34]. This means that $\mu$ is given by the formula: $\mu_{m}(s):=\frac{\bar{\mu} s}{k+s}$, with $k>0$ and $\bar{\mu}>0$. A generalization of this expression is given by the Hill function $\mu(s)=\mu_{m}\left(s^{\beta}\right)$, for some $\beta>0$, that satisfies also Hypothesis H1. In section 5, we also consider the case where $\mu$ is linear: $\mu(s)=m s, m>0$.

Without any loss of generality, we take $s_{i n}=1$ (by doing a change of variable). We consider the set $\mathcal{U}$ of admissible controls as measurable functions $u(\cdot)$ that take values in $U$ :

$$
\mathcal{U}:=\{u: \mathbb{R} \rightarrow U \mid u \text { meas. }\} .
$$

Classically, Hypothesis H1 ensures the existence and uniqueness of solutions of (1.3) for any admissible control and positive time. Since $\mu(\cdot)$ is defined only for $s \geq 0$, only trajectories contained in the positive orthant are meaningful. Straightforwardly, one can check that the domain

$$
E:=\left\{\left(s_{1}, s_{2}\right) \in \mathbb{R}_{+}^{2} \mid 0 \leq s_{2} \leq s_{1} \leq 1\right\},
$$

is forwardly invariant for any $u(\cdot) \in \mathcal{U}$. Notice that the line segment

$$
L:=\{1\} \times[0,1],
$$

that lies on the boundary of $E$ is also invariant. Moreover, the minimal time problem with initial conditions in $L$ (and $\bar{s} \in L$ ) is a one-dimensional problem and is straightforward to solve. From now on, we consider targets in the interior of the domain $E$, and we shall consider in the following initial conditions in the set $\mathcal{D}$ defined by:

$$
\mathcal{D}:=E \backslash L \text {. }
$$

Notice that $\mathcal{D}$ is neither open nor closed. Next, we assume that the maximum dilution rate is large enough to compete the growth of the species on the substrate, that is $u_{\max }$ larger than $\mu(1)$. This assumption is required for the controllability of the variable $s_{1}$ (otherwise there exist values of $s_{1}$ for which we have $\dot{s}_{1} \geq 0$ for any control $u_{1}$ ). Without any loss of generality we can also take $u_{\max }=1$ (one can always re-normalize time and $\mu(\cdot)$ of a factor $\left.1 / u_{\max }\right)$ :

Hypothesis H2. $u_{\max }=1$ and $\mu(s)<1$ for any $s \in[0,1]$.

For any admissible control $u(\cdot)$, we shall denote, for convenience, $s\left(\cdot, s^{0}, u(\cdot)\right)$ the forward solution of (1.3) with $s(0)=s^{0}$, and $s^{-}\left(\cdot, s^{0}, u(\cdot)\right)$ the backward solution, that is $s^{-}\left(t, s^{0}, u(\cdot)\right)=s\left(-t, s^{0}, u(-\cdot)\right)$ for any $t \geq 0$. We shall also denote $\gamma^{+}\left(s^{0}, u(\cdot)\right)$ and $\gamma^{-}\left(s^{0}, u(\cdot)\right)$ the positive and negative semi-orbits of the dynamics with control $u(\cdot)$. Similarly, we denote by $\gamma\left(s^{0}, u(\cdot)\right)$ the orbit of the dynamics with control $u(\cdot)$. We also denote by $\partial A$ the boundary of a set $A \subset \mathcal{D}$ and $\operatorname{Int} A$ its interior. 
In order to apply Pontryagin's Principle, we recall the definition the reachable set from a point $s^{0}$, see [3]. Given $\bar{s} \in \mathcal{D}, s^{0} \in \mathcal{D}$, and $u \in \mathcal{U}$, we define $t_{s^{0}}(u) \in[0,+\infty]$ as the first entry time into the target point $\bar{s}$, that is:

$$
t_{s^{0}}(u):=\inf \{t \geq 0 \mid s(t)=\bar{s}\},
$$

where $s(t):=s\left(t, s^{0}, u(t)\right)$. Now, the optimal control problem that we consider can be stated as follows. Let $T: \mathcal{D} \rightarrow[0,+\infty]$ be the minimal time function:

$$
T\left(s^{0}\right):=\inf _{u \in \mathcal{U}} t_{s^{0}}(u) .
$$

Our aim is to find an optimal control $u$ for any $s^{0} \in \mathcal{D}$ attaining the infimum in (2.1). The controllability set is defined as the set of starting points from which the system can reach the target in a finite time $t>0$ :

$$
\mathcal{R}:=\left\{s^{0} \in \mathcal{D} \mid T\left(s^{0}\right)<+\infty\right\} .
$$

Given $s^{0} \in \mathcal{R}$, the existence of an optimal control for (2.1) is straightforward using Filippov's Theorem. Therefore, we can apply the Pontryagin's Maximum Principle for (2.1) to characterize optimal trajectories. The Hamiltonian

$$
H=H\left(s_{1}, s_{2}, \lambda_{0}, \lambda_{1}, \lambda_{2}, u_{1}, u_{2}\right)
$$

associated to (1.3) is defined by:

$$
H:=-\lambda_{1} \mu\left(s_{1}\right)\left(1-s_{1}\right)-\lambda_{2} \mu\left(s_{2}\right)\left(1-s_{2}\right)+\lambda_{0}+\lambda_{1}\left(1-s_{1}\right) u_{1}+\lambda_{2}\left(s_{1}-s_{2}\right) u_{2} .
$$

Pontryagin's Maximum principle can be stated as follows. Let $u:=\left(u_{1}, u_{2}\right)$ an optimal control and $s:=\left(s_{1}, s_{2}\right)$ the associated trajectory. There exists $t_{f}>0, \lambda_{0} \leq 0$ and $\lambda:\left[0, t_{f}\right] \rightarrow \mathbb{R}^{2}$ satisfying $\left(\lambda_{0}, \lambda(\cdot)\right) \neq 0$ and the adjoint equations $\dot{\lambda}=-\frac{\partial H}{\partial s}$, that is:

$$
\left\{\begin{array}{l}
\dot{\lambda}_{1}=\lambda_{1}\left[\mu^{\prime}\left(s_{1}\right)\left(1-s_{1}\right)-\mu\left(s_{1}\right)+u_{1}\right]-\lambda_{2} u_{2}=\lambda_{1}\left[\nu^{\prime}\left(s_{1}\right)+u_{1}\right]-\lambda_{2} u_{2}, \\
\dot{\lambda}_{2}=\lambda_{2}\left[\mu^{\prime}\left(s_{2}\right)\left(1-s_{2}\right)-\mu\left(s_{2}\right)+u_{2}\right]=\lambda_{2}\left[\nu^{\prime}\left(s_{2}\right)+u_{2}\right] .
\end{array}\right.
$$

Moreover, we have the maximization condition:

$$
u(t) \in \arg \max _{0 \leq \omega_{2} \leq \omega_{1} \leq 1} H\left(s_{1}(t), s_{2}(t), \lambda_{0}, \lambda_{1}(t), \lambda_{2}(t), \omega_{1}, \omega_{2}\right),
$$

for a.e. $t \in\left[0, t_{f}\right]$. An extremal trajectory is a sextuplet

$$
\left(s_{1}(\cdot), s_{2}(\cdot), \lambda_{0}, \lambda_{1}(\cdot), \lambda_{2}(\cdot), u(\cdot)\right)
$$

satisfying (1.3)-(2.3)-(2.4). We say that an extremal is normal whenever $\lambda_{0} \neq 0$ (in that case, we can take $\lambda_{0}=-1$ ). In the case where $\lambda_{0}=0$, we say that the extremal trajectory is abnormal. The Hamiltonian is zero along an extremal trajectory (as $t_{f}$ is free), thus we obtain:

$$
(2.5)-\lambda_{1} \mu\left(s_{1}\right)\left(1-s_{1}\right)-\lambda_{2} \mu\left(s_{2}\right)\left(1-s_{2}\right)+\lambda_{0}+\lambda_{1}\left(1-s_{1}\right) u_{1}+\lambda_{2}\left(s_{1}-s_{2}\right) u_{2}=0 .
$$

It is convenient to consider the switching function $\phi$ defined by:

$$
\phi:=\lambda_{1}\left(1-s_{1}\right)+\lambda_{2}\left(s_{1}-s_{2}\right) .
$$

REMARK 2.1. As we will see, it is not necessary to introduce switching functions associated to both controls $u_{1}$ and $u_{2}$ in order to find a control law from (2.4). We will only use the switching function given by (2.6). This choice is motivated from the dependency between $u_{1}$ and $u_{2}$, see (1.2). The function $\phi$ will be also used in Section 4. 
2.2. Definition of the set $\mathcal{C}(\bar{s})$. We first give asymptotic properties of forward and backward solutions for the extreme controls $(0,0)$ and $(1,1)$ that will be useful in the definition of the set $\mathcal{C}(\bar{s})$. Recall that we have set $\nu(s):=\mu(s)(1-s)$.

Lemma 2.1. Take an initial state $s^{0} \in \mathcal{D} \backslash\{(0,0)\}$.

- For the control $u=(0,0)$, set $s(t)=\left(s_{1}(t), s_{2}(t)\right):=s\left(t, s^{0}, u(t)\right)$. Then, one has:

$$
\lim _{t \rightarrow+\infty} s_{1}(t)=\lim _{t \rightarrow+\infty} s_{2}(t)=0 \text { with } \lim _{t \rightarrow+\infty} \frac{s_{2}(t)}{s_{1}(t)}<+\infty
$$

and

$$
\lim _{t \rightarrow-\infty} s_{1}(t)=\lim _{t \rightarrow-\infty} s_{2}(t)=1 \text { with } \lim _{t \rightarrow-\infty} \frac{1-s_{2}(t)}{1-s_{1}(t)}<+\infty,
$$

where the limits of the ratios only depend on $s^{0}$.

- For the control $u=(1,1)$ set $s(t)=\left(s_{1}(t), s_{2}(t)\right):=s\left(t, s^{0}, u(t)\right)$. Then, one has:

$$
\lim _{t \rightarrow+\infty} s_{1}(t)=\lim _{t \rightarrow+\infty} s_{2}(t)=1 \text { with } \lim _{t \rightarrow+\infty} \frac{1-s_{2}(t)}{1-s_{1}(t)}=+\infty .
$$

In backward time, $s(\cdot)$ leaves $\mathcal{D}$ at a finite time $t_{e}$ with $s_{1}\left(-t_{e}\right)=s_{2}\left(-t_{e}\right)$ or $s_{2}\left(-t_{e}\right)=0$.

Proof. For $u=(0,0)$ the $s_{1}$ and $s_{2}$ sub-systems are decoupled with

$$
\dot{s}_{i}=-\nu\left(s_{i}\right),
$$

$\nu(0)=\nu(1)=0$ and $\nu(s)>0$ for $s \in(0,1)$. Thus, $s_{i}^{0} \notin\{0,1\}$ implies that one has necessarily

$$
\lim _{t \rightarrow+\infty} s_{i}(t)=0 \text { and } \lim _{t \rightarrow-\infty} s_{i}(t)=1,
$$

for $i=1,2$. From (2.7), one obtains that the following property is fulfilled for any $t$

$$
\int_{s_{1}^{0}}^{s_{1}(t)} \frac{d \sigma}{\nu(\sigma)}=\int_{s_{2}^{0}}^{s_{2}(t)} \frac{d \sigma}{\nu(\sigma)} \Longrightarrow \int_{s_{2}^{0}}^{s_{1}^{0}} \frac{d \sigma}{\nu(\sigma)}=\int_{s_{2}(t)}^{s_{1}(t)} \frac{d \sigma}{\nu(\sigma)},
$$

with

$$
\lim _{t \rightarrow+\infty} \int_{s_{2}(t)}^{s_{1}(t)} \frac{d \sigma}{\nu(\sigma)}=\lim _{t \rightarrow+\infty} \int_{s_{2}(t)}^{s_{1}(t)} \frac{d \sigma}{\nu^{\prime}(0) \sigma}=\frac{1}{\nu^{\prime}(0)} \lim _{t \rightarrow+\infty} \log \left(\frac{s_{2}(t)}{s_{1}(t)}\right)
$$

and

$$
\lim _{t \rightarrow-\infty} \int_{s_{2}(t)}^{s_{1}(t)} \frac{d \sigma}{\nu(\sigma)}=\lim _{t \rightarrow-\infty} \int_{s_{2}(t)}^{s_{1}(t)} \frac{d \sigma}{\nu^{\prime}(1)(\sigma-1)}=\frac{1}{\nu^{\prime}(1)} \lim _{t \rightarrow-\infty} \log \left(\frac{1-s_{2}(t)}{1-s_{1}(t)}\right) .
$$

Finally, we obtain that:

$$
\lim _{t \rightarrow+\infty} \frac{s_{2}(t)}{s_{1}(t)}=\exp \left(\int_{s_{2}^{0}}^{s_{1}^{0}} \frac{\nu^{\prime}(0)}{\nu(\sigma)} d \sigma\right), \lim _{t \rightarrow-\infty} \frac{1-s_{2}(t)}{1-s_{1}(t)}=\exp \left(\int_{s_{2}^{0}}^{s_{1}^{0}} \frac{\nu^{\prime}(1)}{\nu(\sigma)} d \sigma\right)
$$


which shows that the limit of the two ratios are finite and depend only on $s^{0}$ as was to be proved.

For $u=(1,1)$ the dynamics of the $s_{1}$ sub-system is

$$
\dot{s}_{1}=\bar{G}\left(s_{1}\right),
$$

where $\bar{G}\left(s_{1}\right):=\left(1-s_{1}\right)\left(1-\mu\left(s_{1}\right)\right)$. As $\mu\left(s_{1}\right)<1$ (see Hypothesis H2), one has then clearly $s_{1}(t) \rightarrow 1$ when $t \rightarrow+\infty$. The $s_{2}$ sub-system can be seen as an asymptotic autonomous dynamics with the same limiting dynamics in forward time:

$$
\dot{s}_{2}=G\left(t, s_{2}\right),
$$

where $G\left(t, s_{2}\right):=-\mu\left(s_{2}\right)\left(1-s_{2}\right)+\left(s_{1}(t)-s_{2}\right)$ satisfies $\left|G\left(t, s_{2}\right)-\bar{G}\left(s_{2}\right)\right| \rightarrow 0$ for any $s_{2} \in(0,1]$ when $t$ goes to $+\infty$. The trajectory being bounded, we deduce that $s_{2}(t)$ tends also to 1 when $t \rightarrow+\infty$, see e.g. [28]. Consider the ratio $r(t)=\left(1-s_{2}(t)\right) /(1-$ $\left.s_{1}(t)\right)$ whose time derivative satisfies

$$
\dot{r}=\left(\mu\left(s_{2}(t)\right)-\mu\left(s_{1}(t)\right)\right) r+1 .
$$

If $r(\cdot)$ is bounded, say by $M>0$, there exists $T>0$ such that $\left[\mu\left(s_{1}(t)\right)-\mu\left(s_{2}(t)\right)\right] M<$ $\alpha<1$ for any $t>T$ as $s_{1}-s_{2}$ is non-negative and tends to zero. Then one should have $\dot{r}(t)>(1-\alpha)>0$ for $t>T$, leading to a contradiction. So we conclude that $r$ tends to $+\infty$. In backward time, the solution $s_{1}(\cdot)$ of $(2.8)$ clearly goes to negative values. So the trajectory has to leave the domain $\mathcal{D}$ in finite time with decreasing $s_{1}$. This is only possible through the parts of the boundary $s_{1}=s_{2}$ or $s_{2}=0$.

REMARK 2.2. The previous Lemma implies that the semi-orbit $\gamma^{+}\left(s^{0},(1,1)\right)$ is below $\gamma^{-}\left(s^{0},(0,0)\right)$ in a neighborhood of the point $(1,1)$. Indeed, both associated trajectories converge to $(1,1)$, and the comparison follows from the limit of the two ratios $\frac{1-s_{1}}{1-s_{2}}$ when the point $s=\left(s_{1}, s_{2}\right)$ goes to $(1,1)$. This important property is used in particular in section 4.1.

Consider now the target point $\bar{s}$ as an initial condition. According to Lemma 2.1, there exists an exit time $t_{e}$ of the domain $\mathcal{D}$ backward in time with constant control $(1,1)$. We then define the function $p(\cdot)$ on the interval $\left[-t_{e},+\infty\right)$ as

$$
p(\tau):=\mid \begin{array}{ll}
s^{-}(-\tau, \bar{s},(1,1)), & \tau \in\left[-t_{e}, 0\right) \\
s^{-}(\tau, \bar{s},(0,0)), & \tau \in[0,+\infty)
\end{array}
$$

If the exit time is such that $s_{1}^{-}\left(t_{e}, \bar{s},(1,1)\right)=s_{2}^{-}\left(t_{e}, \bar{s},(1,1)\right)$, we set $I=\left[-t_{e},+\infty\right)$. Otherwise, we extend $p(\cdot)$ on $\left[-t_{e}-s_{1}^{-}\left(t_{e}, \bar{s},(1,1)\right),-t_{e}\right)$ as

$$
p(\tau)=\left(\tau+t_{e}+s_{1}^{-}\left(t_{e}, \bar{s},(1,1)\right), 0\right)
$$

and set $I=\left[-t_{e}-s_{1}^{-}\left(t_{e}, \bar{s},(1,1)\right),+\infty\right)$. Recall that we have $\dot{p}_{1}>0$ over $I$, so the support of the curve $p(\cdot)$ can be parameterized as a function $s_{1} \longmapsto s_{2}\left(s_{1}\right)$. Hence, we can define the set $\mathcal{C}(\bar{s})$ as the epigraph of the function $p$ restricted to the domain $\mathcal{D}$ :

$$
\mathcal{C}(\bar{s}):=\left\{s \in \mathcal{D} \mid s_{1}=p_{1}(\tau), s_{2} \geq p_{2}(\tau), \tau \in I\right\}
$$

Remark 2.3. The line segment $L$ does not belong to $\mathcal{C}(\bar{s})$. 
According to Lemma 2.1, the set $\mathcal{C}(\bar{s})$ has two possible shapes, depending on which part of the boundary of $\mathcal{D}$ the semi-orbit $\gamma^{-}(\bar{s},(1,1))$ leaves the domain $\mathcal{D}$. To distinguish these two shapes, it is convenient to introduce the curve $\mathcal{F}$ defined by $\mathcal{F}:=\gamma^{+}((0,0),(1,1))$. From Lemma $2.1, \mathcal{F}$ provides a partition of the domain $\mathcal{D}$ into two sub-domains. Cauchy-Lipschitz Theorem implies that if $\bar{s}$ is above $\mathcal{F}$ (resp. below $\mathcal{F}$ ), then $\partial \mathcal{C}(\bar{s})$ intersects the first diagonal (resp. the axis $s_{2}=0$ ). On pictures of Fig. 1, one can see an illustration of the two cases.
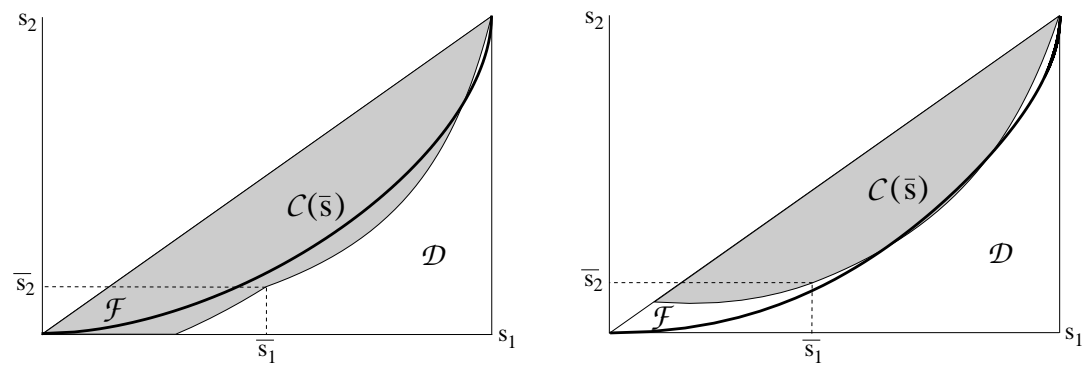

Figure 1. Picture of the set $\mathcal{C}(\bar{s})$ for $\mu(s)=s$. In brown the curve $\mathcal{F}$. On the left : the negative semi-orbit of $\bar{s}=(1,0.3)$ with $u_{1}=u_{2}=1$ intersects the axis $s_{2}=0$. On the right: the negative semi-orbit of $\bar{s}=(0.9,0.32)$ with $u_{1}=u_{2}=1$ intersects the first diagonal.

3. Optimality result in $\mathcal{C}(\bar{s})$. In this section, we provide an optimality result for initial conditions in $\mathcal{C}(\bar{s})$. Let us consider the set of semi-orbits that reach $\bar{s}$ with $u_{1} \equiv 1$ or $u_{2} \equiv 0$ :

$$
\mathcal{S}(\bar{s}):=\mathcal{S}^{-}(\bar{s}) \cup \mathcal{S}^{+}(\bar{s})
$$

with

$$
\mathcal{S}^{-}(\bar{s}):=\bigcup_{v(\cdot) \in \mathcal{V}} \gamma^{-}(\bar{s},(1, v(\cdot))) \cap \mathcal{D}, \quad \mathcal{S}^{+}(\bar{s}):=\bigcup_{v(\cdot) \in \mathcal{V}} \gamma^{-}(\bar{s},(v(\cdot), 0)) \cap \mathcal{D},
$$

where $\mathcal{V}$ is the set of measurable functions $v$ that take values on $[0,1]$ :

$$
\mathcal{V}:=\{u:[0,+\infty) \rightarrow[0,1] \mid u \text { meas. }\} .
$$

This set possesses the following nice property.

Proposition 3.1. For any $\bar{s} \in \mathcal{D}$, one has $\mathcal{C}(\bar{s})=\mathcal{S}(\bar{s})$.

Proof. Let us first prove that $\mathcal{C}(\bar{s}) \subset \mathcal{S}(\bar{s})$. Take $s^{0} \in \mathcal{C}(\bar{s})$, and consider the trajectory $s(\cdot):=s\left(\cdot, s^{0},(1,0)\right)$. If there exists $t_{0} \geq 0$ such that $s\left(t_{0}\right)=\bar{s}$, then we have $s^{0}=s^{-}\left(t_{0}, \bar{s},(1,0)\right) \in \mathcal{S}(\bar{s})$ as was to be proved. Now, we suppose that for any $t \geq 0$, we have $s(t) \neq \bar{s}$. Having $0<s_{2}(0)<s_{1}(0)$ and $s_{2}(\cdot)$ decreasing, $s(\cdot)$ has to leave $\mathcal{D}$ at a finite time $t_{0}$ : either it intersects at time $t_{0}$ the semi-orbit $\gamma^{-}(\bar{s},(1,1))$ (case a), or it intersects at time $t_{0}$ the semi-orbit $\gamma^{-}(\bar{s},(0,0))$ (case b).

Case $a$. Let $t_{1}>0$ be such that $\bar{s}=s\left(t_{1}, s\left(t_{0}\right),(1,1)\right)$. Then, let us define a control $u:\left[0, t_{0}+t_{1}\right] \rightarrow U$ by $u=(1,1)$ on $\left[0, t_{1}\right]$ and $u=(1,0)$ on $\left[t_{1}+t_{0}\right]$. By construction, we have $s^{-}\left(t_{1}+t_{0}, \bar{s}, u\right)=s^{0}$, and $u$ is of the form $u=\left(1, u_{2}\right)$ with $u_{2} \in \mathcal{V}$, and the result follows.

Case b. Let $t_{1}^{\prime}>0$ be such that $\bar{s}=s\left(t_{1}^{\prime}, s\left(t_{0}\right),(0,0)\right)$. Then, let us define a control $\tilde{u}:\left[0, t_{0}+t_{1}^{\prime}\right] \rightarrow U$ by $\tilde{u}=(0,0)$ on $\left[0, t_{1}^{\prime}\right]$ and $\tilde{u}=(1,0)$ on $\left[t_{1}^{\prime}+t_{0}\right]$. By 
construction, we have $s^{-}\left(t_{1}^{\prime}+t_{0}, \bar{s}, \tilde{u}\right)=s^{0}$, and $\tilde{u}$ is of the form $\tilde{u}=\left(u_{1}, 0\right)$ with $u_{1} \in \mathcal{V}$, and the result follows.

Let us now prove that $\mathcal{S}(\bar{s}) \subset \mathcal{C}(\bar{s})$. Take $s^{0} \in \mathcal{S}(\bar{s})$, and assume first that $s^{0}=s^{-}\left(t_{0}, \bar{s},\left(1, u_{2}\right)\right)$ with $u_{2} \in \mathcal{V}$ and $t_{0} \in\left[0, t_{e}\right)$ where $t_{e}$ is the exit time of $\mathcal{D}$. For $t \in\left[0, t_{e}\right)$, set $\tilde{s}(t):=s^{-}\left(t, \bar{s},\left(1, u_{2}\right)\right)$, and $\hat{s}(t):=s^{-}(t, \bar{s},(1,1))$. One can straightforwardly prove that $\tilde{s}_{1}(t)=\hat{s}_{1}(t)$ and $\tilde{s}_{2}(t) \geq \hat{s}_{2}(t)$ for all $t \in\left[0, t_{e}\right)$.

Case $a$. If $s_{1}^{-}\left(t_{e}, \bar{s},\left(1, u_{2}\right)\right)=s_{2}^{-}\left(t_{e}, \bar{s},\left(1, u_{2}\right)\right)$, one has $\tilde{s}_{1}\left(t_{0}\right)=\hat{s}_{1}\left(t_{0}\right), \tilde{s}_{2}\left(t_{0}\right) \geq \hat{s}_{2}\left(t_{0}\right)$, $\hat{s}\left(t_{0}\right) \in \partial \mathcal{C}(\bar{s})$, and as $t_{0} \leq t_{e}$, we get that $\tilde{s}\left(t_{0}\right) \in \mathcal{C}(\bar{s})$.

Case b. If $s_{2}^{-}\left(t_{e}, \bar{s},(1,1)\right)=0$, one has $\tilde{s}_{1}\left(t_{0}\right)=\hat{s}_{1}\left(t_{0}\right), \tilde{s}_{2}\left(t_{0}\right) \geq \max \left(0, \hat{s}_{2}\left(t_{0}\right)\right)$, $\left(\hat{s}_{1}\left(t_{0}\right), \max \left(0, \hat{s}_{2}\left(t_{0}\right)\right)\right) \in \partial \mathcal{C}(\bar{s})$ so that $t_{0} \leq t_{e}$ implies $\tilde{s}\left(t_{0}\right) \in \mathcal{C}(\bar{s})$.

Suppose now that $s^{0}=s^{-}\left(t_{0}, \bar{s},\left(u_{1}, 0\right)\right)$ with $u_{1} \in \mathcal{V}$ and $t_{0} \in\left[0, t_{e}\right)$ where $t_{1}$ is the exit time of $\mathcal{D}$. For $t \in\left[0, t_{e}\right)$, set $\tilde{s}(t):=s^{-}\left(t, \bar{s},\left(u_{1}, 0\right)\right), \check{s}(t):=s^{-}(t, \bar{s},(0,0))$, and $\hat{s}(t):=s^{-}(t, \bar{s},(1,0))$ Similarly as in the previous case, we obtain:

$$
\tilde{s}_{2}(t)=\check{s}_{2}(t)=\hat{s}_{2}(t), \quad \text { and } \quad \hat{s}_{1}(t) \leq \tilde{s}_{1}(t) \leq \check{s}_{1}(t), \quad \forall t \in\left[0, t_{e}\right) .
$$

Notice that $\bar{s}_{2}>0$ so that at the exit time $t_{e}$, we necessarily have $\tilde{s}_{2}\left(t_{1}\right)=\tilde{s}_{1}\left(t_{e}\right)$. Moreover, combining the fact that $\left(\check{s}_{1}\left(t_{0}\right), \check{s}_{2}\left(t_{0}\right)\right) \in \partial \mathcal{C}(\bar{s})$ and that $\left(\hat{s}_{1}(t), \hat{s}_{2}(t)\right) \in$ $\mathcal{C}(\bar{s})$ for all $t \in\left[0, t_{e}\right)$, we obtain that $\tilde{s}\left(t_{0}\right) \in \mathcal{C}(\bar{s})$ as was to be proved. $\square$

Consider the particular semi-orbit in $\mathcal{S}(\bar{s})$ :

$$
\Gamma:=\gamma^{-}(\bar{s},(1,0)) \cap \mathcal{D},
$$

and notice that it can be parameterized as a curve $s_{1} \mapsto s_{2}=\alpha\left(s_{1}\right)$ because we have $\dot{s}_{1}<0$ on this semi-orbit. One has the following property.

Lemma 3.2. Take $\bar{s} \in \mathcal{D}$. The subset $\mathcal{S}^{-}(\bar{s})$, resp. $\mathcal{S}^{+}(\bar{s})$, is the set of points $s=\left(s_{1}, s_{2}\right) \in \mathcal{S}(\bar{s})$ that satisfy $s_{2} \leq \alpha\left(s_{1}\right)$, resp. $s_{2} \geq \alpha\left(s_{1}\right)$. Furthermore $\mathcal{S}^{-}(\bar{s}) \cap$ $\mathcal{S}^{+}(\bar{s})=\Gamma$.

Proof. Let $\tilde{s}(\cdot):=s^{-}(\cdot, \bar{s},(1,0))$, and consider a backward trajectory $s(\cdot):=$ $s^{-}\left(\cdot, \bar{s},\left(1, u_{2}\right)\right)$ with $u_{2} \in \mathcal{V}$. One has clearly $s_{1}(t)=\tilde{s}_{1}(t)$ and $s_{2}(t)<\tilde{s}_{2}(t)$ for any time $t$. Consequently, any trajectory in $\mathcal{C}(\bar{s})$ with $u_{1} \equiv 1$ is below $\Gamma$. Similarly, any trajectory in $\mathcal{C}(\bar{s})$ with $u_{2} \equiv 0$ is above $\Gamma$. Clearly, $\Gamma$ is the only semi-orbit of $\mathcal{S}(\bar{s})$ that belongs to $\mathcal{S}^{-}(\bar{s})$ and $\mathcal{S}^{+}(\bar{s})$.

Proposition 3.1 shows that for any $s^{0} \in \mathcal{C}(\bar{s})$, there exists an admissible control steering $s^{0}$ to $\bar{s}$ in finite time, provided that $\bar{s} \in \mathcal{D}$. We shall now give a characterization of the optimal trajectories.

Proposition 3.3. Let $\bar{s} \in \mathcal{D}$. For any initial state $s^{0} \in \mathcal{C}(\bar{s})$, the optimal trajectories belong to $\mathcal{C}(\bar{s})$, and the optimal feedbacks $u[\cdot]: s \longmapsto u[s] \in U$ are given by:

- for $s \in \mathcal{S}^{-}(\bar{s}) \backslash \Gamma, u_{1}[s]=1$ and $u_{2}[s]$ takes any value in $[0,1]$,

- for $s \in \mathcal{S}^{+}(\bar{s}) \backslash \Gamma, u_{2}[s]=0$ and $u_{1}[s]$ takes any value in $[0,1]$,

- for $s \in \Gamma, u[s]=(1,0)$.

Furthermore, the value function in $\mathcal{C}(\bar{s})$ is:

$$
T\left(s^{0}\right)=\max \left\{\int_{s_{1}^{0}}^{\overline{s_{1}}} \frac{d \sigma}{(1-\mu(\sigma))(1-\sigma)}, \int_{s_{2}^{0}}^{\overline{s_{2}}} \frac{d \sigma}{-\mu(\sigma)(1-\sigma)}\right\} .
$$


Proof. Let $s^{0} \in \mathcal{C}(\bar{s})$. We know from Proposition 3.1 that there exists a control $u \in \mathcal{U}$ of the form $\left(1, u_{2}(\cdot)\right)$ or $\left(u_{1}(\cdot), 0\right)$ and a time $t_{u} \in[0,+\infty[$ such that

$$
s\left(t_{u}, s^{0}, u\right)=\bar{s} .
$$

First, suppose that $u$ is of the form $\left(1, u_{2}\right)$ and set $s(\cdot):=s\left(\cdot, s^{0}, u\right)$. Therefore one has

$$
\dot{s_{1}}=\left(1-\mu\left(s_{1}\right)\right)\left(1-s_{1}\right)
$$

hence $s_{1}(\cdot)$ is increasing and:

$$
s_{1}(t)<\bar{s}_{1}, \quad \forall t \in\left[0, t_{u}\right)
$$

We show that $t_{u}$ is optimal. If not, there exists $\hat{u} \in \mathcal{U}$ such that $s\left(T\left(s^{0}\right), s^{0}, \hat{u}\right)=\bar{s}$ with $T\left(s^{0}\right)<t_{u}$. Set $\hat{s}(\cdot):=s\left(\cdot, s^{0}, \hat{u}\right)$. As $\hat{u}_{1} \leq 1$ and $\hat{s}_{1}(0)=s_{1}(0)=s_{1}^{0}$, we obtain by $(1.3)$ :

$$
\hat{s}_{1}(t) \leq s_{1}(t), \quad \forall t \in[0,+\infty) .
$$

Therefore, combining (3.3) and (3.4), we get:

$$
\bar{s}_{1}=\hat{s}_{1}\left(T\left(s^{0}\right)\right) \leq s_{1}\left(T\left(s^{0}\right)\right)<\bar{s}_{1}
$$

which is a contradiction, hence $t_{u}=T\left(s^{0}\right)$. Let us now explicit $t_{u}=T\left(s^{0}\right)$. One has:

$$
t_{u}=\int_{0}^{t_{u}} d t=\int_{0}^{t_{u}} \frac{\dot{s}_{1}(t)}{\left(1-\mu\left(s_{1}(t)\right)\right)\left(1-s_{1}(t)\right)} d t=\int_{s_{1}^{0}}^{\bar{s}_{1}} \frac{d \sigma}{(1-\mu(\sigma))(1-\sigma)}
$$

Finally let us show that

$$
\int_{s_{2}^{0}}^{\bar{s}_{2}} \frac{d \sigma}{-\mu(\sigma)(1-\sigma)} \leq \int_{s_{1}^{0}}^{\bar{s}_{1}} \frac{d \sigma}{(1-\mu(\sigma))(1-\sigma)}
$$

Remark that $1 /(-\mu(s)(1-s))<0$, for any $s \in(0,1)$. Therefore if $s_{2}^{0} \leq \bar{s}_{2}$, one has $\int_{s_{2}^{0}}^{\bar{s}_{2}} \frac{d \sigma}{-\mu(\sigma)(1-\sigma)} \leq 0$ and the result is obvious. Let us now suppose that $s_{2}^{0}>\bar{s}_{2}$ and set $\varphi$ as the solution of the Cauchy problem:

$$
\left\{\begin{array}{l}
\dot{\varphi}=-\mu(\varphi)(1-\varphi) \\
\varphi(0)=s_{2}^{0}
\end{array}\right.
$$

Then, $\varphi$ is decreasing and converges to zero when $t$ tends to $+\infty$. Thus, there exists $t_{0} \in[0,+\infty)$ such that $\varphi\left(t_{0}\right)=\bar{s}_{2}$. Therefore, one has

$$
\int_{s_{2}^{0}}^{\bar{s}_{2}} \frac{d \sigma}{-\mu(\sigma)(1-\sigma)}=\int_{\varphi(0)}^{\varphi\left(t_{0}\right)} \frac{d \sigma}{-\mu(\sigma)(1-\sigma)}=\int_{0}^{t_{0}} d t=t_{0} .
$$

Moreover, as $u_{2} \geq 0$ and $s_{2}(0)=\varphi(0)$ one has by (1.3) that $\varphi(t) \leq s_{2}(t)$ for all $t \in\left[0,+\infty\right.$. Consequently, as $\varphi$ is decreasing with $\varphi\left(t_{0}\right)=s_{2}\left(t_{u}\right) \geq \varphi\left(t_{u}\right)$, one has $t_{0} \leq t_{u}$, which by (3.6) and (3.8) gives (3.7). Thus we have proved the expression (3.2) of the value function for such initial condition. 
From Proposition 3.1 and Lemma 3.2, any trajectory with initial condition $s^{0}$ and control $\left(1, u_{2}\right)$ that is below $\Gamma$ is also above $\gamma^{-}(\bar{s},(1,1))$. Consequently, any such trajectory reaches $\bar{s}$ in finite time, and stays in $\mathcal{C}(\bar{s})$ until this time. As all these trajectories have exactly the same map $t \mapsto s_{1}(t)$, we deduce that $\bar{s}$ is reached exactly at the same time $t_{u}$. This shows the optimality of all the trajectories with control $u_{1}=1$ as long as $s$ is below $\Gamma$.

Now, we investigate the case where $u$ is of the form $\left(u_{1}, 0\right)$. We set $s(\cdot)=$ : $s\left(\cdot, s^{0}, u\right)$. We have that $s_{2}(\cdot)$ is decreasing and therefore $s_{2}^{0}>\bar{s}_{2}$. Similarly as in the previous case, we can show that $v\left(s^{0}\right)=t_{u}$ (using the fact that $s_{2}^{0}>\bar{s}_{2}$ ), and that $t_{u}=\int_{s_{2}^{0}}^{\bar{s}_{2}} \frac{d \sigma}{-\mu(\sigma)(1-\sigma)}$. Finally, we show that:

$$
\int_{s_{1}^{0}}^{\bar{s}_{1}} \frac{d \sigma}{(1-\mu(\sigma))(1-\sigma)} \leq \int_{s_{2}^{0}}^{\bar{s}_{2}} \frac{d \sigma}{-\mu(\sigma)(1-\sigma)} .
$$

First, if $s_{1}^{0} \geq \bar{s}_{1}$, then $\int_{s_{1}^{0}}^{\bar{s}_{1}} \frac{d \sigma}{(1-\mu(\sigma))(1-\sigma)} \leq 0$ and (3.9) is obvious. If now $s_{1}^{0}<\bar{s}_{1}$, we consider $\varphi$ as the solution of the following Cauchy problem:

$$
\left\{\begin{array}{l}
\dot{\varphi}=(1-\mu(\varphi))(1-\varphi), \\
\varphi(0)=s_{1}^{0} .
\end{array}\right.
$$

As $\varphi$ is increasing, there exists $t_{0}>0$ such that $\int_{s_{1}^{0}}^{\bar{s}_{1}} \frac{d \sigma}{(1-\mu(\sigma))(1-\sigma)}=t_{0}$ with $\varphi\left(t_{0}\right)=\bar{s}_{1}$. Moreover, as $\varphi$ satisfies the same equation as $s_{1}$ with the constant control equal to 1 in place of $u_{1}$, we obtain $\varphi\left(t_{0}\right)=s_{1}\left(t_{u}\right)=\bar{s}_{1} \leq \varphi\left(t_{u}\right)$, so that $t_{0} \leq t_{u}$ (as $\varphi$ is increasing). We conclude similarly as in the first case.

Finally, if $s \in \Gamma$, the optimal trajectory has to fulfill the properties of both former cases, that is $u_{1}[s]=1$ and $u_{2}[s]=0$.

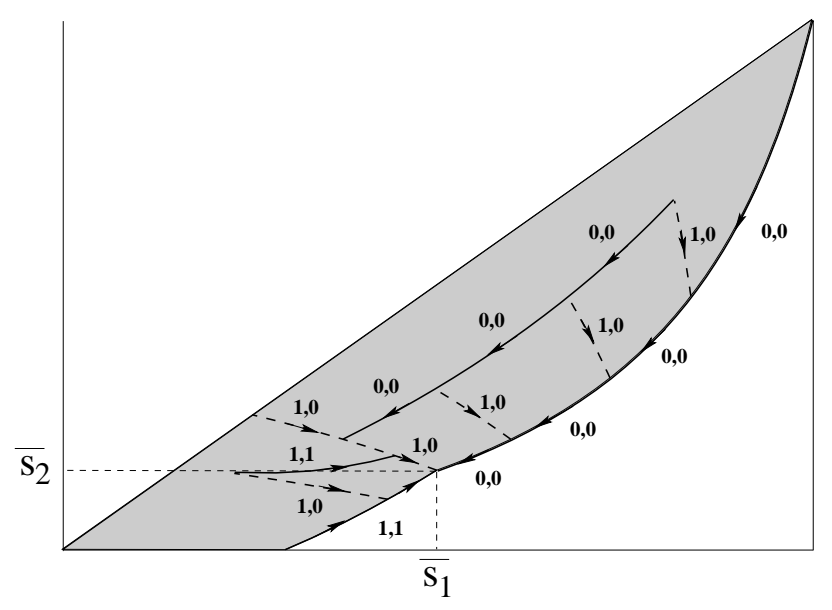

Figure 2. Picture for $\bar{s}=(1,0.3)$. When the initial condition $s^{0}$ is below $\Gamma$, there exist infinitely many trajectories in $\mathcal{S}^{-}(\bar{s})$ steering $s^{0}$ to $\bar{s}$ in the same time $T\left(s^{0}\right)$ with $u_{1} \equiv 1$. When $s^{0}$ is above $\Gamma$, there exist infinitely many trajectories in $\mathcal{S}^{+}(\bar{s})$ steering $s^{0}$ to $\bar{s}$ in the same time $T\left(s^{0}\right)$ with $u_{2} \equiv 0$.

Fig. 2 depicts the infinity of optimal trajectories from an initial state $s^{0} \in \mathcal{C}(\bar{s}) \backslash \Gamma$ (see Proposition 3.3). Notice that when $s^{0} \in \mathcal{S}^{-}(\bar{s})$, resp. $s^{0} \in \mathcal{S}^{+}(\bar{s})$, optimal 
trajectories are such that $\lambda_{1} \equiv 0$, resp. $\lambda_{2} \equiv 0$ (where $\lambda$ is the corresponding adjoint vector). This can be easily proved via the Pontryagin's Maximum Principle, and corresponds to $s^{0}$ such that $\partial_{1} T\left(s^{0}\right)=0$ or $\partial_{2} T\left(s^{0}\right)=0$ showing that different initial conditions could have the same optimal cost, thus explaining the infinity of optimal trajectories. From a practical viewpoint, practitioners may use the most convenient choice of controls away from the curve $\Gamma$, for instance taking $u_{1}=u_{2}=u$ with $u=1$ when $s \in \mathcal{S}^{-}(\bar{s}) \backslash \Gamma$, and $u=0$ when $s \in \mathcal{S}^{+}(\bar{s}) \backslash \Gamma$. When the trajectory touches the curve $\Gamma$, the control $(1,0)$ has to be used.

Remark 3.1. Proposition 3.3 can be also proved using Hamilton-Jacobi Equation in the viscosity sense (taking into account that the value function $T(\cdot)$ is nondifferentiable on the curve $\Gamma$ ).

4. Optimality result outside $\mathcal{C}(\bar{s})$. In this part, we provide optimal trajectories for initial conditions in $\mathcal{D} \backslash \mathcal{C}(\bar{s})$ which allows to conclude on the optimal synthesis of the problem. Theorem 4.17 is our main result. First, we show that for initial conditions outside the set $\mathcal{C}(\bar{s})$, an optimal control satisfies $u_{1}=u_{2}$.

Proposition 4.1. Let us consider an initial condition $s^{0} \in \mathcal{D} \backslash \mathcal{C}(\bar{s})$, and assume that $s^{0} \in \mathcal{R}$ (recall (2.2)). Then, an optimal control $u \in \mathcal{U}$ steering $s^{0}$ to $\bar{s}$ satisfies $u_{1}=u_{2}$ a.e. and $\lambda_{2}>0$.

Proof. First, assume that we have $\lambda_{2} \equiv 0$. From (2.3), $\lambda_{1}$ is of constant sign (either positive or negative). If $\lambda_{1}>0$, then $u_{1}=1$ a.e. and $u_{2}$ is any control taking values in $[0,1]$. Proposition 3.1 implies that $s^{0} \in \mathcal{C}(\bar{s})$ which is a contradiction. If $\lambda_{1}<0$, then we have $u_{1}=0$ a.e., thus $u_{2}=0$ a.e. implying that $s^{0} \in \partial \mathcal{C}\left(s^{0}\right)$ which again gives a contradiction.

Now, let us investigate the case where $\lambda_{2}<0$. If there exists a time interval $\left[t_{1}, t_{2}\right]$ where $u_{2}>0$, then, we obtain a contradiction in the maximization condition (2.4) by comparing this control to the constant one equal to zero. This implies that $u_{2}=0$ a.e., and from (2.3), $\lambda_{1}$ is of constant sign. First, if $\lambda_{1}>0$, then $u_{1}=1$ a.e. by (2.4), and we see that $s^{0} \in \Gamma \subset \mathcal{C}(\bar{s})$ implying a contradiction. Now, if $\lambda_{1}<0$, then $u_{1}=0$ a.e., and both controls are constantly equal to zero, thus $s^{0} \in \partial \mathcal{C}\left(s^{0}\right)$ implying a contradiction. If $\lambda_{1} \equiv 0$, then $u_{1}$ is any measurable control taking values within $[0,1]$. Using Proposition 3.1 which characterizes $\mathcal{C}(\bar{s})$, we obtain that necessarily $s^{0} \in \mathcal{C}(\bar{s})$ which is a contradiction.

It follows that we have $\lambda_{2}>0$ as was to be proved. We now show that this implies $u_{1}=u_{2}$ for an optimal trajectory. From the inequality $u_{2} \leq u_{1}$, we obtain that

$$
\lambda_{1}\left(1-s_{1}\right) u_{1}+\lambda_{2}\left(s_{1}-s_{2}\right) u_{2} \leq \phi u_{1} .
$$

If we combine this inequality together with (2.4), we obtain the following: if $\phi>0$, then the maximum in the Hamiltonian is achieved for $u_{2}=u_{1}=1$. If $\phi<0$, then it is achieved for $u_{1}=u_{2}=0$. If $\phi=0$, then we have

$$
\lambda_{1}\left(1-s_{1}\right) u_{1}+\lambda_{2}\left(s_{1}-s_{2}\right) u_{2}=\lambda_{2}\left(s_{1}-s_{2}\right)\left(u_{2}-u_{1}\right)
$$

and we see using $u_{2} \leq u_{1}$ that the maximum is obtained when $u_{1}=u_{2}$. Therefore, we have obtained that in the three previous cases $\phi>0, \phi<0$ and $\phi=0$, we have $u_{1}=u_{2}$. Hence, for $s^{0} \in \mathcal{D} \backslash \mathcal{C}(\bar{s})$, an optimal control satisfies $u_{1}=u_{2}$ and $\lambda_{2}(\cdot)>0$ as was to be proved. 
We are now in position to study the minimal time problem outside $\mathcal{C}(\bar{s})$ using the theory of affine control systems in the plane with one input. We can write (1.3) as:

$$
\dot{s}=f(s)+v g(s), v \in \mathcal{V},
$$

where $f, g$ are the two vector fields defined by:

$$
f(s):=-\left[\begin{array}{c}
\nu\left(s_{1}\right) \\
\nu\left(s_{2}\right)
\end{array}\right], g(s):=\left[\begin{array}{c}
1-s_{1} \\
s_{1}-s_{2}
\end{array}\right],
$$

and $u_{1}=u_{2}=v \in \mathcal{V}$ with $\mathcal{V}$ given by (3.1). It is standard to introduce the noncontrollability set $\Delta_{0}$ (also called collinearity set sometimes) see [5, 6, 25] by:

$$
\Delta_{0}:=\left\{\left(s_{1}, s_{2}\right) \in \mathcal{D} \mid \operatorname{det}(f(s), g(s))=0\right\},
$$

and the singular arc $\Delta_{S A}$ by:

$$
\Delta_{S A}:=\left\{\left(s_{1}, s_{2}\right) \in \mathcal{D} \mid \operatorname{det}(g(s),[f, g](s))=0\right\},
$$

where $[f, g]$ denotes the Lie bracket of $f$ and $g$, see e.g. [25]. Next, we define $\Delta_{0}^{+}$(resp. $\left.\Delta_{0}^{-}\right)$as the set of points of $\mathcal{D}$ such that $\operatorname{det}(f(s), g(s))>0(\operatorname{resp} . \operatorname{det}(f(s), g(s))<0)$. Similarly, we define $\Delta_{S A}^{ \pm}$. A simple computation shows that we have:

$$
\begin{array}{r}
\operatorname{det}(g(s),[f, g](s))=\left[1-s_{1}\right]\left[\left(\mu\left(s_{2}\right)-\mu\left(s_{1}\right)-\mu^{\prime}\left(s_{1}\right)\left(s_{1}-s_{2}\right)\right)\left(1-s_{1}\right)\right. \\
\left.+\mu^{\prime}\left(s_{2}\right)\left(1-s_{2}\right)\left(s_{1}-s_{2}\right)\right] .
\end{array}
$$

As the point $(1,1)$ is an equilibrium of $(1.3)$, the singular arc is given by the implicit equation

$$
\left[\mu\left(s_{2}\right)-\mu\left(s_{1}\right)-\mu^{\prime}\left(s_{1}\right)\left(s_{1}-s_{2}\right)\right]\left(1-s_{1}\right)+\mu^{\prime}\left(s_{2}\right)\left(1-s_{2}\right)\left(s_{1}-s_{2}\right)=0 .
$$

The function $\phi$ is the switching function for the minimal time control problem governed by (4.1), and a computation shows that:

$$
\dot{\phi}=\mu^{\prime}\left(s_{1}\right)\left(1-s_{1}\right) \phi+\lambda_{2} \frac{\operatorname{det}(g(s),[f, g](s))}{1-s_{1}} .
$$

The next proposition gives properties of the set $\Delta_{0}$.

Proposition 4.2. There exists a continuous mapping $s_{1} \in[0,1] \longmapsto \zeta\left(s_{1}\right)$ of class $C^{1}$ over $[0,1)$ such that $\Delta_{0}$ is the graph of the restriction of $\zeta$ over $[0,1)$. Moreover, the following properties hold:

(i) $\zeta(0)=0, \zeta(1)=1$ and $\zeta$ is increasing over $[0,1]$.

(ii) $\lim _{s_{1} \rightarrow 1} \frac{1-\zeta\left(s_{1}\right)}{1-s_{1}}=+\infty$.

Proof. Let us consider the $C^{1}$-mapping

$$
\rho:\left(s_{1}, s_{2}\right) \in \mathcal{D} \longmapsto \rho\left(s_{1}, s_{2}\right):=-\mu\left(s_{1}\right)\left(s_{1}-s_{2}\right)+\mu\left(s_{2}\right)\left(1-s_{2}\right)
$$

so that $\rho\left(s_{1}, s_{2}\right)=0$ iff $s \in \Delta_{0}$. For $s \in \mathcal{D}$, we have:

$$
\frac{\partial \rho}{\partial s_{2}}\left(s_{1}, s_{2}\right)=\mu\left(s_{1}\right)-\mu\left(s_{2}\right)+\mu^{\prime}\left(s_{2}\right)\left(1-s_{2}\right)>0 .
$$


Hence, we can apply the implicit function Theorem for every $s \in[0,1)$ which provides the existence of a function $\zeta:[0,1) \rightarrow \mathcal{D}$ such that $s \in \Delta_{0} \backslash\{(1,1)\}$ if and only if $s_{2}=\zeta\left(s_{1}\right)$. Moreover, we have $\rho(0,0)=0$ implying $\zeta(0)=0$. Now, the derivative of $\zeta$ w.r.t. $s_{1}$ is given by:

$$
\zeta^{\prime}\left(s_{1}\right)=\frac{\mu^{\prime}\left(s_{1}\right)\left(s_{1}-s_{2}\right)+\mu\left(s_{1}\right)}{\mu\left(s_{1}\right)-\mu\left(s_{2}\right)+\mu^{\prime}\left(s_{2}\right)\left(1-s_{2}\right)},
$$

where $s_{2}=\zeta\left(s_{1}\right)$. One has clearly $\zeta^{\prime}\left(s_{1}\right)>0$ on $(0,1)$. Hence $\zeta$ is increasing over $(0,1)$. As we have $\rho(1,1)=0$, we have $\zeta\left(s_{1}\right) \rightarrow 1$ whenever $s_{1} \rightarrow 1$. Hence, we can extend $\zeta$ continuously on $[0,1]$ by letting $\zeta(1)=1$ which shows (i). Moreover, if we let $s_{1} \rightarrow 1$ in (4.5), we obtain that $\zeta^{\prime}\left(s_{1}\right) \rightarrow+\infty$, which shows (ii).

Finding an optimal synthesis highly depends on the position of the target with respect to $\Delta_{0}$ and $\Delta_{S A}$. In the following, we call steady-state singular point (see [6]) any point $s^{\star} \in \mathcal{D}$ such that:

$$
s^{\star} \in \Delta_{0} \cap \Delta_{S A},
$$

and we assume throughout this section that there exist such points. The existence of such points plays a major role in our study.

REMARK 4.1.

(i) Such points are equilibriums of the dynamics restricted to the singular arc. In fact, along a singular (normal) extremal trajectory defined over a time interval $\left[t_{1}, t_{2}\right]$, the adjoint vector satisfies $\lambda(t) \cdot f(s(t))=1$ and $\lambda(t) \cdot g(s(t))=0, t \in\left[t_{1}, t_{2}\right]$, where $g(s) \neq 0$ for every $s \in \mathcal{D}$, which shows that it cannot reach $s^{\star}$ in finite time. This implies that if the singular arc is admissible, then $s^{\star}$ is never reached by a singular (normal) trajectory in finite time.

(ii) Even if $s^{\star}$ cannot be reached in finite time, an extremal singular trajectory can be locally time minimizing. In other words, the singular arc can be hyperbolic according to the classification given in [5].

(iii) If we consider two different trajectories steering a point $s^{0}$ to $\bar{s}$ (which intersect only at points $s^{0}$ and $\bar{s}$ ), then we cannot apply the clock form argument globally if the domain enclosed to the union of these curves intersects $\Delta_{0}$. We remind that this tool is based on Green's Theorem (see [27, 5, 25]) and gives a direct method to compare the cost of two trajectories that do not intersect $\Delta_{0}$.

Let us recall the following (general) result, see Lemma 13 in [6] that will be useful in order to find the optimal synthesis.

Lemma 4.3. Consider two vector fields $f, g: \mathbb{R}^{2} \rightarrow \mathbb{R}^{2}$ of class $C^{1}$, and suppose that $f$ and $g$ are non-zero over $\Delta_{0}$. Let us define Tan as the set of points of $\mathcal{D} \backslash\{(0,0)\}$ where the dynamics (4.1) is tangent to $\Delta_{0}$ for any $u \in[0,1]$. Then Tan is non-empty, and we have:

$$
\operatorname{Tan}=\Delta_{0} \cap \Delta_{S A} .
$$

Proof. For sake of completeness, we provide a different proof of this result. At any $s \in \Delta_{0} \backslash(\{(0,0)\} \cup\{(1,1)\})$, the vector fields $f$ and $g$ are non-zero and collinear, so that one can write $f(s)=\alpha g(s)$ where $\alpha \neq 0$ is a scalar (that depends on $s$ ). The 
dynamics is then tangent to $\Delta_{0}$ for any control $v$ if $f(s)$ or $g(s)$ belongs to the tangent cone of $\Delta_{0}$ at $s$, that is defined as

$$
T_{\Delta_{0}}(s):=\left\{\delta \in \mathbb{R}^{2} \mid \operatorname{det}(f(s+h \delta), g(s+h \delta))=O\left(h^{2}\right)\right\} .
$$

Having $f(s) \in T_{\Delta_{0}}(s)$ amounts to write

$$
\begin{aligned}
& \operatorname{det}\left(f(s)+h \partial_{s} f(s) \cdot f(s)+O\left(h^{2}\right), g(s)+h \partial_{s} g(s) \cdot f(s)+O\left(h^{2}\right)\right)=O\left(h^{2}\right), \\
\Leftrightarrow & \operatorname{det}(f(s), g(s))+h \operatorname{det}\left(f, \partial_{s} g(s) \cdot f(s)\right)+h \operatorname{det}\left(\partial_{s} f(s) \cdot f(s), g(s)\right)=0, \\
\Leftrightarrow & \operatorname{det}\left(\alpha g(s), \partial_{s} g(s) \cdot f(s)\right)-\operatorname{det}\left(g(s), \alpha \partial_{s} f(s) \cdot g(s)\right)=0, \\
\Leftrightarrow & \operatorname{det}(g(s),[f, g](s))=0,
\end{aligned}
$$

that is $s \in \Delta_{S A}$.

This result applies in particular on the vector fields (4.2). Throughout the rest of the paper, we make the following assumption that is essential in our study.

Hypothesis H3. There exists a unique steady-state singular point $s^{\star} \in \mathcal{D}$.

REMARK 4.2 .

(i) Hypothesis H3 is satisfied whenever $\mu$ is of Monod type or linear (see section 5). Thanks to numerical simulations, we conjecture that H3 holds in the case where $\mu:[0,1] \rightarrow \mathbb{R}_{+}$is a concave increasing function of class $C^{2}$ such that $\mu(0)=0$. Simple counter-examples show that this result no longer holds whenever $\mu$ is not concave.

(ii) The concavity of growth functions appears to be a natural hypothesis for modeling a saturation effect under large values of substrate concentrations.

For $s \in[0,1]$, we denote by $\tau(s)$ the unitary tangent vector of $\Delta_{0}$ at point $s$. The next Lemma provides the direction of the drift in (4.1) along the curve $\Delta_{0}$, that is parameterized as in Proposition 4.2, and is quite useful in the following.

Lemma 4.4. Let $s \in \Delta_{0}$. Then, we have:

$$
s \in \Delta_{S A}^{+} \Rightarrow \operatorname{det}(f(s), \tau(s))>0, s \in \Delta_{S A}^{-} \Rightarrow \operatorname{det}(f(s), \tau(s))<0 .
$$

Proof. As $s \in \Delta_{0}$, we have $\mu\left(s_{1}\right)\left(s_{1}-s_{2}\right)=\mu\left(s_{2}\right)\left(1-s_{2}\right)$. From (4.5), we can write the vector $\tau(s)$ as $\tau(s)=\kappa\left(\begin{array}{c}1 \\ \zeta^{\prime}\left(s_{1}\right)\end{array}\right)$, where $\kappa>0$ is a coefficient to normalize $\tau(s)$ (that depends on $s$ ). Using that $s_{1}>s_{2}$, the sign of $\operatorname{det}(f(s), \tau(s))$ is given by the quantity defined by:

$$
\begin{array}{r}
\alpha(s):=-\mu\left(s_{1}\right)\left(1-s_{1}\right)\left[\mu\left(s_{1}\right)+\mu^{\prime}\left(s_{1}\right)\left(s_{1}-s_{2}\right)\right]+\mu\left(s_{2}\right)\left(1-s_{2}\right)\left(\mu\left(s_{1}\right)-\mu\left(s_{2}\right)\right. \\
\left.+\mu^{\prime}\left(s_{2}\right)\left(1-s_{2}\right)\right) .
\end{array}
$$

Using the fact that $s \in \Delta_{0}$, we find:

$$
\begin{aligned}
\frac{\alpha(s)}{\mu_{1}(s)}=\left(1-s_{1}\right)\left(\mu\left(s_{2}\right)\right. & \left.-\mu\left(s_{1}\right)-\mu^{\prime}\left(s_{1}\right)\left(s_{1}-s_{2}\right)\right)-\left(1-s_{1}\right) \mu\left(s_{2}\right) \\
& +\left(s_{1}-s_{2}\right)\left(\mu\left(s_{1}\right)-\mu\left(s_{2}+\mu^{\prime}\left(s_{2}\right)\left(1-s_{2}\right)\right) .\right.
\end{aligned}
$$

Now, let us take a point $s \in \Delta_{S A}^{-}$. Then, (4.3) implies that:

$$
\begin{aligned}
\frac{\alpha(s)}{\mu_{1}(s)} & <-\left(1-s_{1}\right) \mu\left(s_{2}\right)+\left(s_{1}-s_{2}\right)\left(\mu\left(s_{1}\right)-\mu\left(s_{2}\right)\right) \\
& \left.=-\left(1-s_{1}\right) \mu\left(s_{2}\right)+\left(1-s_{2}\right) \mu\left(s_{2}\right)-\left(s_{1}-s_{2}\right) \mu\left(s_{2}\right)\right)=0 .
\end{aligned}
$$


where the equality is obtained using the fact that $s \in \Delta_{0}$. It follows that for $s \in \Delta_{S A}^{-}$, we have $\alpha(s)<0$, thus $\operatorname{det}(f(s), \tau(s))<0$ which proves the result. When $s \in \Delta_{S A}^{+}$, we use the same computation and the reverse inequality. This proves the lemma.

The colinear directions of the dynamics with controls $(0,0)$ and $(1,1)$ are depicted on Fig. 3 as an illustration of the statement of Lemma 4.4.

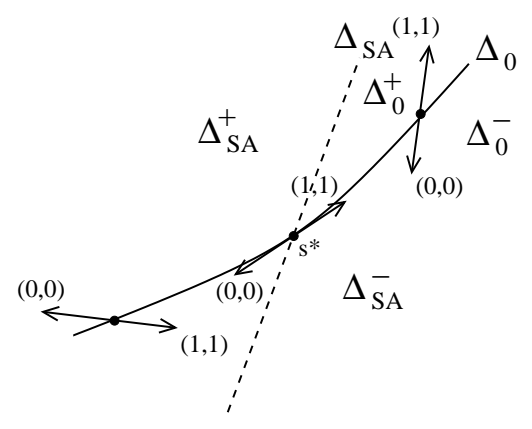

FIGURE 3. Directions of the dynamics with controls $(0,0)$ and $(1,1)$ for states on the curve $\Delta_{0}$.

The next proposition is the basis of the optimality results of this section and is based on Lemma 4.3.

Proposition 4.5. Let us take $\bar{s} \in \mathcal{D}$. Then, either $s^{\star} \in \mathcal{C}(\bar{s})$ or $\mathcal{C}(\bar{s}) \subset \Delta_{0}^{+}$.

Proof. Recall that the boundary of $\mathcal{C}(\bar{s})$ is the union of semi-orbits $\gamma^{-}(\bar{s},(0,0))$ and $\gamma^{-}(\bar{s},(1,1))$. Let us suppose that $s^{\star} \notin \mathcal{C}(\bar{s})$, and assume by contradiction that there exists a point $\hat{s} \in \partial \mathcal{C}(\bar{s}) \cap \Delta_{0}$. We have several cases.

First case. $\hat{s} \in \Delta_{S A} \bar{S}^{-} \cap \gamma^{-}(\bar{s},(0,0))$. Then, from Lemma 4.4, the boundary of $\mathcal{C}(\bar{s})$ with $v=0$ necessarily intersects $\Delta_{0}$ in $\Delta_{S A}^{+}$. This would give $s^{\star} \in \mathcal{C}(\bar{s})$ which is a contradiction.

Second case. $\hat{s} \in \Delta_{S A}^{-} \cap \gamma^{-}(\bar{s},(1,1))$. Then, from Lemma 4.4, the boundary of $\mathcal{C}(\bar{s})$ with $v=1$ or $v=0$ cannot intersect $\Delta_{0}$ in the set $\Delta_{S A}^{-}$. This would give $s^{\star} \in \mathcal{C}(\bar{s})$ which is a contradiction.

The study of the case where the intersection point $\hat{s}$ is in $\Delta_{S A}^{+}$is similar to the previous case. Hence, we have obtained the result.

Given a target point $\bar{s} \in \mathcal{D}$, one should determine whenever $\bar{s}$ is such that $s^{\star} \in$ $\mathcal{C}(\bar{s})$ or not. To do so, we introduce a curve $\Lambda$ passing through $s^{\star}$ and that provides a partition of the set $\mathcal{D}$ :

$$
\Lambda:=\gamma^{+}\left(s^{\star},(0,0)\right) \cup \gamma^{+}\left(s^{\star},(1,1)\right) .
$$

This curve is depicted on Fig. 5 and 8 in the case of a linear growth function. It satisfies the following properties.

LEMMA 4.6.

(i) There exists a mapping $q:(0,1) \rightarrow(0,1)$ of class $C^{1}$ with $q^{\prime}>0$ and such that the curve $\Lambda$ coincides with the graph of $q$.

(ii) The curve $\Lambda$ is such that $\Lambda \cap \Delta_{0}=\left\{s^{\star}\right\}$ and we have

$$
\Lambda \backslash\left\{s^{\star}\right\} \subset \Delta_{0}^{+} .
$$


Proof. For proving (i), notice that $\Lambda$ is defined via the controls $(0,0)$ and $(1,1)$. The parameterization follows from Lemma 2.1 and the fact that $f$ and $f+g$ are collinear at point $s^{\star}$.

Let us prove (ii). First, we show that $\gamma^{+}\left(s^{\star},(0,0)\right) \backslash\left\{s^{\star}\right\}$ is contained in $\Delta_{0}^{+}$. Suppose now that the semi-orbit $\gamma^{+}\left(s^{\star},(0,0)\right)$ intersects $\Delta_{0}$ at some point $s \neq s^{\star}$ from $\Delta_{0}^{+}$into $\Delta_{0}^{-}$. Then we have a contradiction from Lemma 4.4. Suppose now that $\gamma^{+}\left(s^{\star},(0,0)\right)$ intersects $\Delta_{0}$ at some point $s \neq s^{\star}$ from $\Delta_{0}^{-}$into $\Delta_{0}^{+}$. Then, there exists a point $\check{s}$ which is above $\gamma^{+}\left(s^{\star},(0,0)\right)$ and below $\Delta_{0}$. Consider now the semi-orbit $\gamma^{-}(\check{s},(0,0))$. By Cauchy-Lipschitz Theorem, this curve cannot intersect $\gamma^{+}\left(s^{\star},(0,0)\right)$. It follows that there exists an intersection point $\check{s}^{\prime} \in \gamma^{-}(\check{s},(0,0)) \cap \Delta_{0}$. This is in contradiction with Lemma 4.4. Hence, $\gamma^{+}\left(s^{\star},(0,0)\right) \backslash\left\{s^{\star}\right\} \subset \Delta_{0}^{+}$.

Similarly, we can use exactly the same arguments to prove that $\gamma^{+}\left(s^{\star},(1,1)\right) \backslash\left\{s^{\star}\right\}$ is contained in $\Delta_{0}^{+}$. This concludes the proof. $\square$

Now, we can define a closed subset of $\mathcal{D}$ as follows:

$$
A:=\left\{s=\left(s_{1}, s_{2}\right) \in \mathcal{D} \cup\{(1,1)\} \mid s_{2} \geq q\left(s_{1}\right) \text { and } s_{2} \leq s_{1}\right\},
$$

and its complementary $B:=\mathcal{D} \backslash A$. Notice that the point $(1,1)$ is introduced in the definition of $A$ for the closeness property (see Definition 4.8) and that $\Lambda \cup(1,1)$ is the boundary of $A$. Proposition 4.5 implies the following result.

Corollary 4.7. If $\bar{s} \in \operatorname{Int} A$ then $\mathcal{C}(\bar{s}) \subset \Delta_{0}^{+}$; if $\bar{s} \in \Lambda$ then $s^{\star} \in \partial \mathcal{C}(\bar{s})$; if $\bar{s} \in B$, then $s^{\star} \in \mathcal{C}(\bar{s})$.

To finish this part, we show a semi-permeability property on the curve $\Lambda[7,8,31]$. To do so, let us write the dynamics (1.3) as $\dot{s}=F(s, u)$, where $F: \mathbb{R}^{2} \times \mathbb{R}^{2} \rightarrow \mathbb{R}^{2}$ is given by (1.3). A curve such that each trajectory of the control system may cross this curve in only one direction is called a semipermeable curve. More precisely, we have the following definition.

Definition 4.8. A closed set $C \subseteq \mathbb{R}^{2}$ has a semipermeable boundary for the dynamics $\dot{s}=F(s, u)$ in a neighborhood of $\bar{s} \in \partial C$, if there exists a neighborhood $V$ of $\bar{s}$ such that

$$
\forall s \in \partial C \cap V, \forall p \in N_{C}(s), \inf _{u \in U}\langle F(s, u), p\rangle=0,
$$

where $N_{C}(s)$ is the normal cone to $C$ at point $s$.

Proposition 4.9. The set $A$ has a semi-permeable boundary on the neighborhood of each point $s \in \Lambda$ for the system (1.3), with outward direction.

Proof. Recall that $\Lambda=\gamma^{+}(\bar{s},(0,0)) \cup \gamma^{+}(\bar{s},(1,1))$ and that $\Lambda \subset \Delta_{0}^{+} \cup\left\{s^{\star}\right\}$. First, we consider a point $s=\left(s_{1}, s_{2}\right) \in \gamma^{+}(\bar{s},(0,0))$. The outward normals of $A$ at point $s \in \Lambda$ are of the form $\lambda n(s)$ with $\lambda>0$ and

$$
n(s)=\left(\begin{array}{c}
\mu\left(s_{2}\right)\left(1-s_{2}\right) \\
-\mu\left(s_{1}\right)\left(1-s_{1}\right)
\end{array}\right)
$$

We show that $\inf _{\left(u_{1}, u_{2}\right) \in U}\left\langle F\left(s,\left(u_{1}, u_{2}\right)\right), n(s)\right\rangle=0$. It is clear that $\langle F(s,(0,0)), n(s)\rangle$ is equal to zero, so we have to show that $\left\langle F\left(s,\left(u_{1}, u_{2}\right)\right), n(s)\right\rangle \geq 0$, for all $\left(u_{1}, u_{2}\right) \in U$. Let $\left(u_{1}, u_{2}\right) \in U$. One has:

$$
\left\langle F\left(s,\left(u_{1}, u_{2}\right)\right), n(s)\right\rangle=\left(1-s_{1}\right)\left[u_{1} \mu\left(s_{2}\right)\left(1-s_{2}\right)-u_{2} \mu\left(s_{1}\right)\left(s_{1}-s_{2}\right)\right] .
$$


As $\left(s_{1}, s_{2}\right) \in \Delta_{0}^{+} \cup \Delta_{0}$, we have $\mu\left(s_{2}\right)\left(1-s_{2}\right) \geq \mu\left(s_{1}\right)\left(s_{1}-s_{2}\right)$, implying that $u_{1} \mu\left(s_{2}\right)\left(1-s_{2}\right) \geq u_{2} \mu\left(s_{1}\right)\left(s_{1}-s_{2}\right)$, that is, $\left\langle F\left(s,\left(u_{1}, u_{2}\right)\right), n(s)\right\rangle \geq 0$ as wanted.

Suppose now that $s \in \gamma^{+}(\bar{s},(1,1))$. Then, the outward normals of $A$ at point $s \in \Lambda$ are of the form $\lambda \cdot n(s)$ with $\lambda>0$ and

$$
n(s)=\left(\begin{array}{c}
-\mu\left(s_{2}\right)\left(1-s_{2}\right)+\left(s_{1}-s_{2}\right) \\
\left(\mu\left(s_{1}\right)-1\right)\left(1-s_{1}\right)
\end{array}\right) .
$$

Notice that we have $\langle F(s,(1,1)), n(c(t))\rangle=0$. Let us now take $\left(u_{1}, u_{2}\right) \in U$. One has:

$$
\begin{array}{r}
\left\langle F\left(s,\left(u_{1}, u_{2}\right)\right), n(s)\right\rangle=\left(1-s_{1}\right)\left[\left(1-u_{1}\right)\left(\mu\left(s_{2}\right)\left(1-s_{2}\right)-\mu\left(s_{1}\right)\left(s_{1}-s_{2}\right)\right)\right. \\
\left.+\left(u_{1}-u_{2}\right)\left(s_{1}-s_{2}\right)\left(1-\mu\left(s_{1}\right)\right)\right] .
\end{array}
$$

Now, as $\left(s_{1}, s_{2}\right) \in \Delta_{0}^{+} \cup \Delta_{0}$, we have $\mu\left(s_{1}\right)\left(s_{1}-s_{2}\right) \leq \mu\left(s_{2}\right)\left(1-s_{2}\right)$. Using that $u_{2} \leq u_{1}$ together with $\mu\left(s_{1}\right)<1$, we obtain

$$
\left\langle F\left(s,\left(u_{1}, u_{2}\right)\right), n(s)\right\rangle \geq 0,
$$

which concludes the proof.

REMARK 4.3. Similarly, for the system (1.3), one can also prove that for any $\bar{s} \in A$, the set $\mathcal{C}(\bar{s})$ has a semi-permeable boundary on the neighborhood of each point $s$ such that $s=p(\tau)$ with $\tau \in I$.

Before investigating the optimal synthesis in the case of a target in $A$ or in $B$ (see Corollary 4.7), we study optimality of abnormal trajectories.

4.1. Study of abnormal trajectories. In this section, we prove that the only abnormal optimal trajectories steering a point $s^{0}$ to the target are the semi-orbits $\gamma^{-}(\bar{s},(1,1))$ and $\gamma^{-}(\bar{s},(0,0))$ that are on the boundary of the set $\mathcal{C}(\bar{s})$, see Proposition 4.12 .

Lemma 4.10. Any abnormal extremal trajectory for (1.3) is such that $\lambda_{2}>0$.

Proof. Suppose that $\lambda_{2} \equiv 0$. Then (2.5) implies $\lambda_{1}\left(1-s_{1}\right)\left(u_{1}-\mu\left(s_{1}\right)\right)=0$ which is not possible in view of (2.4). Suppose now that $\lambda_{2}<0$. Then, $\lambda_{1}$ is non-zero and of constant sign from (2.3). If $\lambda_{1}>0$, then we obtain by (2.4)

$$
\lambda_{1}\left(1-s_{1}\right)\left(1-\mu\left(s_{1}\right)\right)-\lambda_{2} \mu\left(s_{2}\right)\left(1-s_{2}\right)=0,
$$

which is a contradiction. Similarly, if $\lambda_{1}<0$, one has by (2.4):

$$
-\lambda_{1}\left(1-s_{1}\right) \mu\left(s_{1}\right)-\lambda_{2} \mu\left(s_{2}\right)\left(1-s_{2}\right)=0,
$$

which is again a contradiction. This ends the proof.

From Proposition 4.1, we obtain that any abnormal extremal trajectory satisfies $u_{1}=u_{2}$. If $s^{0} \in \mathcal{C}(\bar{s})$, then the only abnormal trajectories are $s^{-}(\cdot, \bar{s},(1,1))$ and $s^{-}(\cdot, \bar{s},(0,0))\left(\right.$ as $\left.u_{1}=u_{2}\right)$. So, we can now assume that $s^{0} \notin \mathcal{C}(\bar{s})$. Hence, we deal with only one control $u_{1}=u_{2}=v$. Recall that for an affine system in the plane with one input $v$, then switching points for abnormal trajectories only occur on the set $\Delta_{0}$ (see Proposition 2 in [6], p. 49). The next property will be used repeatedly hereafter. As in Section 3, we use parameterization of orbits w.r.t. $s_{1}$. 
Property 4.1. For any $s^{0}, s^{1} \in \Delta_{0}^{+}$, let us denote $s_{2}=\alpha\left(s_{1}\right)$, resp. $s_{2}=\beta\left(s_{1}\right)$ a parameterization of the orbit $\gamma\left(s^{0},(0,0)\right)$, resp. the semi-orbit $\gamma^{+}\left(s^{1},(1,1)\right)$. If $\alpha\left(s_{1}^{1}\right)>\beta\left(s_{1}^{1}\right)$, then the semi-orbit $\gamma^{+}\left(s^{1},(1,1)\right)$ cannot intersect $\gamma\left(s^{0},(0,0)\right)$ in $\Delta_{0}^{+}$.

Proof. From Lemma 4.4, the semi-orbit $\gamma^{+}\left(s^{1},(1,1)\right)$ is contained in the set $\Delta_{0}^{+}$. For any $s \in \Delta_{0}^{+}$, one has $\operatorname{det}(f(s), f(s)+g(s))>0$. If the conclusion of the property is false, then we would have $\operatorname{det}(f(s), f(s)+g(s)) \leq 0$ at the intersection point $s \in \Delta_{0}^{+}$ between $\gamma^{+}\left(s^{0},(0,0)\right)$ and $\gamma^{+}\left(s^{1},(1,1)\right)$. $\square$

Roughly speaking, a trajectory with the control $(1,1)$ cannot intersect in $\Delta_{0}^{+}$a trajectory with control $(0,0)$ from below (see Fig. 4).

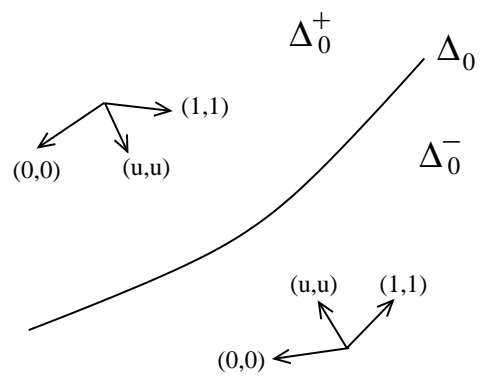

FIGURE 4. Sets of admissible directions for controls $(u, u)$ on both sides of the curve $\Delta_{0}$.

We now show that an abnormal extremal trajectory that has a switching point on $\Delta_{0}$ is not optimal.

Lemma 4.11. Any abnormal extremal trajectory starting at a point $s^{0} \in \mathcal{D} \backslash \mathcal{C}(\bar{s})$ and that has at least one switching point is not optimal.

Proof. Consider an abnormal extremal trajectory starting at some point $s^{0} \in$ $\mathcal{D} \backslash \mathcal{C}(\bar{s})$. If $t_{0}$ is a switching point of such a trajectory, then $s\left(t_{0}\right) \in \Delta_{0}, \phi\left(t_{0}\right)=0$ and we have from (4.4):

$$
\dot{\phi}\left(t_{0}\right)=\frac{\lambda_{2}\left(t_{0}\right)}{1-s_{1}\left(t_{0}\right)} \operatorname{det}\left(g\left(s\left(t_{0}\right)\right),[f, g]\left(s\left(t_{0}\right)\right)\right) .
$$

We now suppose that an abnormal extremal trajectory has a switching point at time $t_{0}$.

First case. $s\left(t_{0}\right) \in \Delta_{S A}^{+}$. From the expression of $\dot{\phi}$ above, we obtain $\dot{\phi}\left(t_{0}\right)>0$. If the trajectory switches from $u=1$ to $u=0$ at time $t_{0}$, then we necessarily have $\dot{\phi}\left(t_{0}\right) \leq 0$ (in fact, one has $\phi(t)>0$ for $t<t_{0}$ ). Thus, we have a contradiction. Hence, the only possibility for the trajectory is to switch in $\Delta_{0} \cap \Delta_{S A}^{+}$from $u=0$ to $u=1$. Then, for any $t>t_{0}$, we have $u=1$ and $s(t) \in \Delta_{0}^{+}$from Lemma 4.4. Property 4.1 implies that the trajectory cannot intersect $\gamma^{-}(\bar{s},(0,0))$. Thus, if the trajectory reaches $\bar{s}$, then $\gamma^{-}(\bar{s},(1,1)) \cap \Delta_{0}^{+}$must coincide with the trajectory for $t \geq t_{0}$. By using Lemma 2.1 (see Remark 2.2) and Property 4.1, we deduce that $s\left(t_{0}\right) \in \mathcal{C}(\bar{s})$, and so is the trajectory for any $t \leq t_{0}$. Now, Proposition 3.3 shows that this abnormal trajectory (which is a concatenation of $u_{1}=u_{2}=0$ and $u_{1}=u_{2}=1$ ) is not optimal.

Second case. $s\left(t_{0}\right) \in \Delta_{S A}^{-}$. This case implies that $\dot{\phi}\left(t_{0}\right)<0$. Similarly, we can exclude a switching point from $u=0$ to $u=1$. We deduce that the trajectory must switch from $u=1$ to $u=0$ at time $t_{0}$. Hence, for $t>t_{0}$, we have $s(t) \in \Delta_{0}^{+}$. By using Property 4.1 and Lemma 4.4, we deduce that the semi-orbit $\gamma^{+}(s(0),(1,1)) \cap \mathcal{D}$ 
is above $\gamma^{+}\left(s\left(t_{0}\right),(0,0)\right)$. Hence, $s\left(t_{0}\right) \in \mathcal{C}(\bar{s})$, and so is the trajectory for any $t \leq t_{0}$, and we conclude as in the previous case.

Third case. $s\left(t_{0}\right) \in \Delta_{S A}$ i.e. $s\left(t_{0}\right)=s^{\star}$. First, suppose that the abnormal trajectory satisfies $u=0$ until $s^{\star}$, then $u=1$. Then we can conclude that it is not optimal by using the arguments in the first case above. Next, if the trajectory satisfies $u=1$ until $s^{\star}$ then $u=0$, we can also conclude that it is not optimal by using the arguments in the second case above.

The previous lemma implies the following result that allows to conclude on the optimality of abnormal trajectories.

Proposition 4.12. The only abnormal optimal trajectories steering a point $s^{0} \in \mathcal{D}$ to $\bar{s}$ are $\gamma^{-}(\bar{s},(1,1))$ and $\gamma^{-}(\bar{s},(0,0))$.

Proof. The semi-orbits $\gamma^{-}(\bar{s},(1,1))$ and $\gamma^{-}(\bar{s},(0,0))$ are the only abnormal trajectories that reach the target without any switching point, and they are optimal thanks to Proposition 3.3.

REMARK 4.4.

(i) This result is in line with the fact that the trajectory associated to an abnormal extremal is on the boundary of the reachable set $\mathcal{R}$ (see [24]).

(ii) When $\partial \mathcal{C}(\bar{s})$ intersects the set $\Delta_{0}$ at some point $s^{a}$ (see for instance Fig. 7 , second picture), then there exists initial conditions in $\mathcal{D}$ for which there exists an abnormal trajectory steering $s^{0}$ to the target $\bar{s}$ and that passes though $s^{a}$. Proposition 4.12 allows to exclude such trajectories from the optimal synthesis.

In the rest of the paper, we can assume that optimal trajectories are normal extremals.

4.2. Study of $\bar{s} \in B$. In this part, we suppose $\bar{s} \in B$, and we show that the optimal strategy outside $\mathcal{C}(\bar{s})$ is of singular type (similarly to the optimal synthesis of minimal time problems for fed-batch bioprocesses with non-monotonic growth, see e.g. $[4,18,29])$. Roughly speaking, the optimal strategy consists in choosing the control that steers the system to the singular arc in minimal time. If the singular arc is reached, then optimal trajectories are singular until reaching $\partial \mathcal{C}(\bar{s})$. Let $s \in \mathcal{D} \backslash \mathcal{C}(\bar{s})$. The singular arc strategy (SAS) is defined by:

$$
u_{1}[s]=u_{2}[s]=v[s]=\mid \begin{array}{cc}
1 & s \in \Delta_{S A}^{-} \\
u_{s}[s] & s \in \Delta_{S A} \\
0 & s \in \Delta_{S A}^{+}
\end{array}
$$

where $u_{s}[\cdot]$ is a singular control such that the solution of (1.3) with $u=u_{s}$ belongs to the set $\Delta_{S A}$. The control $u_{s}[\cdot]$ can be expressed in feedback form:

$$
u_{s}[s(t)]=-\frac{\langle\lambda(t),[f,[f, g]](s(t))\rangle}{\langle\lambda(t),[g,[f, g]](s(t))\rangle}
$$

where the adjoint vector only depends on the state $\lambda(t)=-\frac{-g(s(t))^{\perp}}{\operatorname{det}(f(s(t)), g(s(t)))}$, see e.g. [6].

A possible way to describe optimality properties of singular arcs is to use the notion of turnpike and anti-turnpike proposed in [6] (p.45). We recall briefly this definition adapted to our setting following [6]. 
DeFinition 4.13. A turnpike (resp. anti-turnpike) is a singular arc $S$ that satisfies:

(i) For every $s \in S$, the vectors $f(s)+g(s)$ and $f(s)$ are not tangent to $\Delta_{S A}$ and point to opposite sides of $\Delta_{S A}$.

(ii) For every $s \in S$, we have $\operatorname{det}(g(s),[f, g](s))=0$ and $\operatorname{det}(f(s), g(s)) \neq 0$.

(iii) The mapping $s \longmapsto \theta(s):=-\frac{\operatorname{det}(g(s),[f, g](s))}{\operatorname{det}(f(s), g(s))}$ satisfies $\theta>0$ (resp. $\theta<0$ ) on $\Delta_{S A}^{+}$and $\theta<0$ (resp. $\left.\theta>0\right)$ on $\Delta_{S A}^{-}$.

From Hypothesis H3, the set $\Delta_{S A}$ can be written:

$$
\Delta_{S A}=\Delta_{S A}^{1} \cup \Delta_{S A}^{2} \cup\left\{s^{\star}\right\},
$$

where $\Delta_{S A}^{i}, i=1,2$ is either a turnpike or an anti-turnpike. Next, we assume that the singular arc is admissible.

Hypothesis H4. The singular arc is admissible i.e. $u_{s}[s] \in[0,1]$ for any $s \in$ $\Delta_{S A}$.

This assumption amounts to choose a value of $u_{\max }$ large enough, so that up to a renormalization of the function $\mu$ with a factor $1 / u_{\max }$, the singular arc is always admissible with a control $u_{s} \in[0,1]$. (see Hypothesis H2).

The next Lemma is a direct consequence of the fact that $\Delta_{0} \cap \Delta_{S A}$ is a singleton (which implies properties (ii) and (iii) of Definition 4.13) and of Hypothesis H4, which implies property (i)).

Lemma 4.14. We have $\Delta_{S A}^{1}=\Delta_{S A} \cap \Delta_{0}^{-}$(resp. $\Delta_{S A}^{2}=\Delta_{S A} \cap \Delta_{0}^{+}$) and $\Delta_{S A}^{1}$ (resp. $\Delta_{S A}^{2}$ ) is a turnpike (resp. anti-turnpike).

Equivalently, $\Delta_{S A} \cap \Delta_{0}^{-}$is hyperbolic or time-minimizing and $\Delta_{S A} \cap \Delta_{0}^{+}$is elliptic or time-maximizing (see [15]). This property can be also obtained using the clock form but locally [5]. This means that $s^{\star}$ is an attractive equilibrium for the dynamics (4.1) restricted to $u=u_{s}[s]$ and the set $\Delta_{S A}$. We can now provide an optimal strategy in the set $\mathcal{D} \backslash \mathcal{C}(\bar{s})$.

Proposition 4.15. The optimal strategy steering any point $s^{0} \in \mathcal{D} \backslash \mathcal{C}(\bar{s})$ to $\mathcal{C}(\bar{s})$ is the SAS strategy.

Proof. Let us take a point $s^{0} \in \Delta_{S A}^{+}$. Assume that an optimal trajectory starting at $s=s_{0}$ contains an arc $v=1$ on some time interval $\left[t_{1}, t_{2}\right]$. Then, the trajectory necessarily contains a switching point (otherwise it would not reach the target). So, we can assume that $\phi\left(t_{2}\right)=0$. Let us show that $s\left(t_{2}\right) \in \Delta_{S A}^{+}$. Otherwise, we would have $s(t) \in \Delta_{S A}$ for some $t \leq t_{2}$. As the singular arc is admissible, this is not possible in $\Delta_{S A} \cap \Delta_{0}^{-}$. Similarly, we cannot have $s(t) \in \Delta_{S A} \cap \Delta_{0}^{+}$as we would have $\operatorname{det}(f(s(t)), f(s(t))+g(s(t)))<0$ in contradiction with $s(t) \in \Delta_{0}^{+}$and Property 4.1. Hence the trajectory switches to $v=0$ in $\Delta_{S A}^{+}$. By using (4.4), $\lambda_{2}>0$, we obtain $\dot{\phi}\left(t_{2}\right)>0$. On the other hand, as we have $v=1$ over $\left[t_{1}, t_{2}\right]$, we have $\phi>0$ over $\left[t_{1}, t_{2}\right)$, hence $\dot{\phi}\left(t_{2}\right) \leq 0$ which gives a contradiction. Hence, the optimal trajectory necessarily satisfies $v=0$ until reaching either the singular arc or $\partial \mathcal{C}(\bar{s})$. In the same way, we can show that an optimal trajectory starting at a point $s^{0} \in \Delta_{S A}^{-}$necessarily satisfies $v=1$ until reaching either the singular arc or $\partial \mathcal{C}(\bar{s})$. The same argument as above shows that it is not optimal for a trajectory to leave the singular before reaching the set $\mathcal{C}(\bar{s})$. 
As mentioned in the proof of the previous result, optimal trajectories may not necessarily reach the singular arc. This can happen if $\Delta_{S A} \subset \mathcal{C}(\bar{s})$ (see Fig. 7, third picture). Nevertheless, the singular strategy remains optimal. Notice that the optimal trajectory steering $s^{0}$ to $\bar{s}$ is unique in this case.

4.3. Study of $\bar{s} \in A$. In the case where the target point is above $\Lambda$, we have the following result.

Proposition 4.16. Consider a target point $\bar{s} \in A$. Then, $\mathcal{C}(\bar{s})$ is not reachable from $\mathcal{D} \backslash \mathcal{C}(\bar{s})$.

Proof. First, let us assume that $\bar{s} \in \operatorname{Int} A$ and suppose that there exists a trajectory steering (1.3) from $s^{0} \in \mathcal{D} \backslash \mathcal{C}(\bar{s})$ to the target. If follows that $\mathcal{C}(\bar{s})$ is reachable from $s^{0}$, hence any optimal trajectory starting from the point $s^{0}$ satisfies $u_{1}=u_{2}=v$. From Proposition 4.5, we obtain that $\mathcal{C}(\bar{s}) \subset \Delta_{0}^{+}$. From (2.4), any optimal trajectory necessarily satisfies $v=0, v=1$ or $v=u_{s}$. As $\mathcal{C}(\bar{s}) \subset \Delta_{0}^{+}$, the trajectory cannot reach $\mathcal{C}(\bar{s})$ with the singular arc. Now, the trajectory necessarily has a switching point in $\Delta_{0}^{+}$at some time $t_{0}$, otherwise it would reach $\bar{s}$ with $v=0$ or $v=1$ which means that $s^{0} \in \partial \mathcal{C}\left(s^{0}\right)$ in contradiction with the choice of $s^{0}$. Using the expression of the Hamiltonian and $\phi\left(t_{0}\right)=0$, we find that:

$$
\lambda_{1}\left(t_{0}\right)=\frac{s_{1}-s_{2}}{1-s_{1}} \frac{1}{\rho\left(s_{1}, s_{2}\right)}, \lambda_{2}\left(t_{0}\right)=-\frac{1}{\rho\left(s_{1}, s_{2}\right)},
$$

where $\rho\left(s_{1}, s_{2}\right)=\mu\left(s_{2}\right)\left(1-s_{2}\right)-\mu\left(s_{1}\right)\left(s_{1}-s_{2}\right)$. But, we have $\rho>0$ in $\Delta_{0}^{+}$, hence we obtain that $\lambda\left(t_{2}\right)<0$ which contradicts the fact that $\lambda_{2}>0$ for initial conditions outside of $\mathcal{C}(\bar{s})$. This means that $\bar{s}$ is not reachable from $s^{0}$ which ends the proof.

Now, we have to investigate the case where $\bar{s} \in \Lambda$. Let $s^{0} \in \mathcal{D} \backslash \mathcal{C}(\bar{s})$. If $\bar{s}=s^{\star}$, then from Lemma 4.3 , we know that $\mathcal{C}(\bar{s}) \backslash s^{\star} \subset \Delta_{0}^{+}$. As a singular trajectory cannot reach $s^{\star}$, we can proceed as in the previous case. Assume now that $\bar{s} \in \gamma^{+}(\bar{s},(0,0)) \backslash s^{\star}$. Notice that the part of the boundary of $\mathcal{C}(\bar{s})$ defined with the controls $u_{1}=u_{2}=0$ coincides with $\Lambda$ in the set $\Delta_{S A}^{-}$. Lemma 4.3 implies that the part of the boundary of $\mathcal{C}(\bar{s})$ defined with the controls $u_{1}=u_{2}=1$ does not intersect $\Delta_{0}$. Therefore, $\mathcal{C}(\bar{s}) \backslash s^{\star} \subset \Delta_{0}^{+}$and we can use the same argument as in the case $\bar{s}=s^{\star}$ to show that $\mathcal{C}(\bar{s})$ cannot be reached from $s^{0}$. In the case where $\bar{s} \in \gamma^{+}(\bar{s},(1,1)) \backslash s^{\star}$, we can use a similar reasoning to show that $\mathcal{C}(\bar{s})$ cannot be reached from $s^{0}$. This concludes the proof. $\square$

4.4. Synthesis of the results. The next Theorem is our main result and is a rephrasing of Propositions 3.3, 4.15 and 4.16.

TheORem 4.17. Assume Hypotheses H1, H2, H3 and H4. Let us take a target point $\bar{s} \in \mathcal{D}$. Then, we have the two following cases.

(i) If $\bar{s} \in A$, then $\bar{s}$ is reachable from initial conditions in $\mathcal{C}(\bar{s})$ only.

(ii) If $\bar{s} \notin A$, then $\bar{s}$ is reachable from any initial condition in $\mathcal{D}$.

Moreover, the optimal synthesis is as follows:

- For $s^{0}$ on the boundary of $\mathcal{C}(\bar{s})$, the optimal control is $(1,1)$ if the trajectory is on $\gamma^{-}(\bar{s},(1,1))$, and $(0,0)$ if it is on $\gamma^{-}(\bar{s},(0,0))$.

- For $s^{0}$ in the interior of $\mathcal{C}(\bar{s})$, any control of the form $\left(u_{1}, 0\right)$ or $\left(1, u_{2}\right)$ is optimal, as long as the trajectory does not cross the curve $\Gamma$ or the boundary of $\mathcal{C}(\bar{s})$. On $\Gamma$, the optimal control is $(1,0)$.

- For $s^{0} \in \mathcal{D} \backslash \mathcal{C}(\bar{s})$ (case (ii) only), the optimal control is given by the singular arc strategy $S A S$ until reaching the boundary of $\mathcal{C}(\bar{s})$. 
In case (i) of Theorem 4.17, we can write the value function as:

$$
T\left(s^{0}\right):= \begin{cases}\max \left\{\int_{s_{1}^{0}}^{\overline{s_{1}}} \frac{d \sigma}{(1-\mu(\sigma))(1-\sigma)}, \int_{s_{2}^{0}}^{\overline{s_{2}}} \frac{d \sigma}{-\mu(\sigma)(1-\sigma)}\right\}, & s^{0} \in \mathcal{C}(\bar{s}), \\ +\infty, & s^{0} \in \mathcal{D} \backslash \mathcal{C}(\bar{s}) .\end{cases}
$$

In the second case of Theorem 4.17, we denote by $T_{1}\left(s^{0}\right)$ the time to drive (1.3) from $s^{0}$ to the target $\bar{s}$ by the singular arc strategy until reaching $\mathcal{C}(\bar{s})$ and then the control $(0,0)$ or $(1,1)$ until reaching $\bar{s}$. We can write the value function as:

$$
T\left(s^{0}\right):= \begin{cases}\max \left\{\int_{s_{1}^{0}}^{\overline{s_{1}}} \frac{d \sigma}{(1-\mu(\sigma))(1-\sigma)}, \int_{s_{2}^{0}}^{\overline{s_{2}}} \frac{d \sigma}{-\mu(\sigma)(1-\sigma)}\right\}, & s^{0} \in \mathcal{C}(\bar{s}), \\ T_{1}\left(s^{0}\right), & s^{0} \in \mathcal{D} \backslash \mathcal{C}(\bar{s}) .\end{cases}
$$

In view of the optimal feedback control defining $T_{1}(\cdot)$, its expression is quite hard to obtain. Nevertheless it can be computed numerically.

Fig. 5 depicts the two main cases depending on the target belonging to the set $A$ or not.
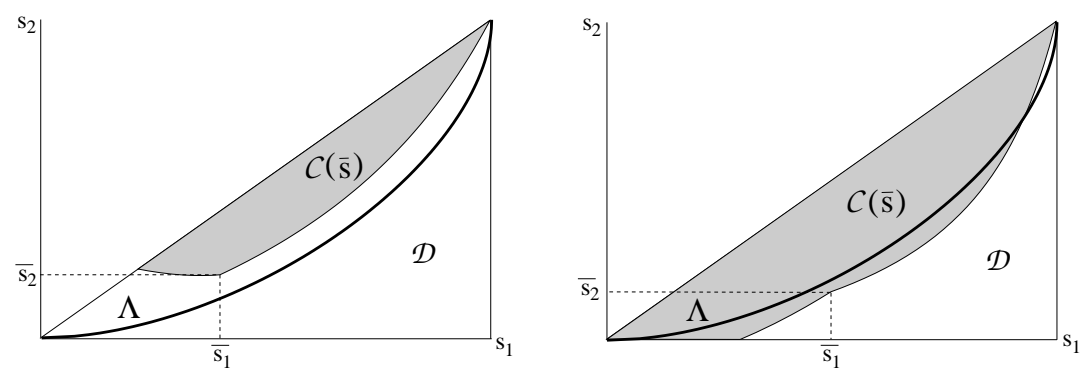

Figure 5. Picture of the set $\mathcal{C}(\bar{s})$ and the curve $\Lambda$ (in thick line). On the left : $\bar{s}=(0.8,0.4)$ belongs to the set $A$. On the right $: \bar{s}=(1,0.3)$ belongs to the set $B$.

4.5. Optimal strategy in $\mathcal{C}(\bar{s})$ with $u_{1}=u_{2}$. We end this section by a comment on the optimal strategy in $\mathcal{C}(\bar{s})$. We have proved that in the set $\mathcal{D} \backslash \mathcal{C}(\bar{s})$, optimal controls $\left(u_{1}, u_{2}\right)$ necessarily satisfy $u_{1}=u_{2}$ which is not the case in the set $\mathcal{C}(\bar{s})$ (see Proposition Proposition 3.3). Therefore, one may wonder what optimal strategy steers the system in minimal time to the target in $\mathcal{C}(\bar{s})$ if we impose $u_{1}=u_{2}$. In view of Proposition 4.15 and using that $\Delta_{S A}$ is time-maximizing in $\Delta_{0}^{+}$, the following result can be obtained similarly.

Proposition 4.18. The optimal feedback steering a point $s^{0} \in \mathcal{C}(\bar{s})$ to the target for the problem with one control $u=u_{1}=u_{2}$ is given by:

$$
u[s]:=\left\{\begin{array}{l}
1, \quad s \in \mathcal{C}(\bar{s}) \backslash \gamma^{-}(\bar{s},(0,0)), \\
0, s \in \gamma^{-}(\bar{s},(0,0)) .
\end{array}\right.
$$

In other words, when both controls are taken equal, the optimal strategy in $\mathcal{C}(\bar{s})$ consists of a bang-bang control $u_{1}=u_{2}=1$ until reaching $\partial \mathcal{C}(\bar{s})$, and then $u_{1}=u_{2}=0$ until reaching $\bar{s}$ if necessary. It is worth noting that this strategy is significantly different than the optimal one with two different controls (in particular for initial conditions on $\Gamma$ where the optimal strategy is $\left.\left(u_{1}, u_{2}\right)=(1,0)\right)$. 


\section{Numerical Simulations.}

5.1. Singular arc when $\mu$ is linear. In this part, we suppose that $\mu(s)=\alpha s$ with $0<\alpha<1$ (see Hypothesis H2). We can prove the following properties on the set $\Delta_{0}$ and the singular arc.

Proposition 5.1.

(i) The set $\Delta_{0}$ is given by the algebraic equation of degree 2:

$$
\Delta_{0}=\left\{\left(s_{1}, s_{2}\right) \in \mathcal{D} \mid-s_{1}\left(s_{1}-s_{2}\right)+s_{2}\left(1-s_{2}\right)=0\right\},
$$

and we have $s_{2}=\zeta\left(s_{1}\right)=\frac{1}{2}\left(s_{1}+1-\sqrt{-3 s_{1}^{2}+2 s_{1}+1}\right), s_{1} \in[0,1]$.

(ii) The set $\Delta_{S A}$ is a line-segment given by

$$
\Delta_{S A}=\left\{\left(s_{1}, s_{2}\right) \in \mathcal{D} \mid s_{2}=2 s_{1}-1\right\} .
$$

(iii) The singular control is given by $u_{s}[s]=2 \alpha\left(1-s_{1}\right)$, and $u_{s}[s] \in[0,1]$ for any $s \in \Delta_{S A}$.

(iv) There exists exactly one steady-state singular point $s^{\star}=\left(\frac{2}{3}, \frac{1}{3}\right)$.

(v) The steady-state singular point is attractive and we have for $s=\left(s_{1}, s_{2}\right) \in$ $\Delta_{S A}$ :

$$
\dot{s}_{1}>0 \text { iff } s_{1} \in\left[\frac{1}{2}, \frac{2}{3}\right) \text { and } \dot{s}_{1}<0 \text { iff } s_{1} \in\left(\frac{2}{3}, 1\right] .
$$

(vi) The adjoint vector along $\Delta_{S A}$ is given by

$$
\lambda_{1}=-\lambda_{2}=-\frac{1}{\alpha\left(1-s_{1}\right)\left(-3 s_{1}+2\right)},
$$

Proof. The proof of (i) and (ii) is straightforward using a symbolic software. Notice that we have $1-s_{1}=s_{1}-s_{2}$ along the singular arc. The expression of $u_{s}$ follows from (1.3) using $\dot{s}_{2}=2 \dot{s}_{1}$ which proves (iii). Now replacing $u_{s}$ into (1.3) gives the closed-loop system:

$$
\left\{\begin{array}{l}
\dot{s}_{1}=\alpha\left(1-s_{1}\right)\left(2-3 s_{1}\right), \\
\dot{s}_{2}=\alpha\left(1-s_{1}\right)\left(1-3 s_{2}\right),
\end{array}\right.
$$

and we obtain (iv)-(v). The proof of (vi) follows by solving $\phi=0$ together with $H=0$ along $\Delta_{S A}$.

This proposition shows in particular that Hypotheses H3 and $\mathrm{H} 4$ are satisfied. This case is illustrated on Fig. 6. The arrows indicate that $s^{\star}$ is attractive. The singular arc in $\Delta_{0}^{-}$(part below the curve $\Delta_{0}$ ) is turnpike (time minimizing) whereas the singular arc in $\Delta_{0}^{+}$(part above $\Delta_{0}$ ) is anti-turnpike (time maximizing). The figures 7 and 8 are an illustration of Theorem 4.17. Several examples of optimal trajectories for initial conditions outside the set $\mathcal{C}(\bar{s})$ are depicted.

5.2. Singular arc when $\mu$ is Monod. In this part, we suppose that the growth function is of Monod type (see [34]): $\mu_{m}(s)=\frac{\bar{\mu} s}{k+s}$, where $k>0, \bar{\mu}>0$ and $\bar{\mu}<1$ (see Hypothesis H2). The situation is quite similar to the linear case, but the expression of $\Delta_{0}, \Delta_{S A}$ and $u_{s}$ are more delicate to obtain. We have used a symbolic software in order to verify the next proposition that provides an explicit expression of $\Delta_{0}$ and $\Delta_{S A}$. 

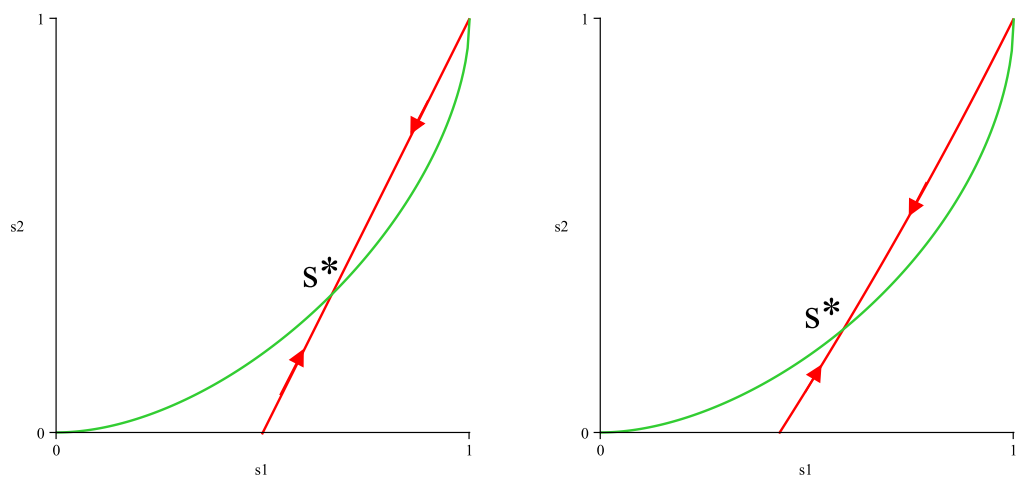

Figure 6. Picture left: plot of the singular arc $\Delta_{S A}$ (dashed line) and of $\Delta_{0}$ (plain line) in the linear case: $\mu(s)=s$. Picture right: plot of the singular arc $\Delta_{S A}$ (dashed line) and of $\Delta_{0}$ (plain line) in the Monod case: $\mu_{m}(s)=\frac{s}{5+s}$.

Proposition 5.2.

(i) The function $\zeta:[0,1] \rightarrow[0,1]$ defining the curve $\Delta_{0}$ is given by:

$$
\zeta\left(s_{1}\right)=-\frac{1}{2 k}\left[s_{1}^{2}-k s_{1}-k-s_{1}+\sqrt{\left(1-s_{1}\right)\left(k+s_{1}-s_{1}^{2}+3 k s_{1}\right)}\right]
$$

with $s_{1} \in(0,1)$.

(ii) There exists $\check{s}_{1} \in(0,1)$ and a $C^{1}$-mapping $\xi:\left[\check{s}_{1}, 1\right) \rightarrow[0,1)$ which is increasing and such that $\left(s_{1}, s_{2}\right) \in \Delta_{S A}$ if and only if $s_{2}=\xi\left(s_{1}\right)$, where :

$$
\xi\left(s_{1}\right):=\frac{1}{2(1-s)}\left[-s_{1}-k^{2}+k s_{1}-3 k+\sqrt{\left(k+s_{1}\right)^{2}(1+k)\left(5+k-4 s_{1}\right)}\right],
$$

and we have $\check{s}_{1}:=\frac{-2 k-1+k \sqrt{4 k^{2}+8 k+5}}{2(1+k)}$.

By using the expression of $\xi$ and $\zeta$, we can check numerically that there exists exactly one singular point $s^{\star}$ (see Fig. 6) and that the singular arc is admissible, hence Hypotheses $\mathrm{H} 3$ and $\mathrm{H} 4$ are satisfied.

6. Conclusion. For this minimal time problem with a set of control inputs with a triangular shape, that is due to the constraint $u_{2} \leq u_{1}$, we have shown the benefit of considering a particular subset $\mathcal{C}(\bar{s})$ of the state space, that is target dependent and presents the following features:

- Outside this set, either the target is non reachable, or the optimal synthesis fulfills $u_{1}=u_{2}$ with a possible singular arc.

- In $\mathcal{C}(\bar{s})$, the extra controllability assumption over control inputs $u_{1}=u_{2}$ (that yields to the inequality $u_{2} \leq u_{1}$ ) leads to an infinity of optimal trajectories, all of them with $u_{2} \neq u_{1}$ (excepted on part of the boundary of the set).

Furthermore, when the the target is reachable from the exterior of $\mathcal{C}(\bar{s})$, the particular cascade structure of the problem leads to a non intuitive feature of some of the optimal trajectories: it consists in rolling far away from the target until reaching the set $\mathcal{C}(\bar{s})$ or a singular arc to be followed until eventually reaching $\mathcal{C}(\bar{s})$, and then come back along the set $\mathcal{C}(\bar{s})$.

The geometric analysis has revealed the role of a semi-permeable curve, that can be easily computed numerically and that indicates to the practitioners whether the 

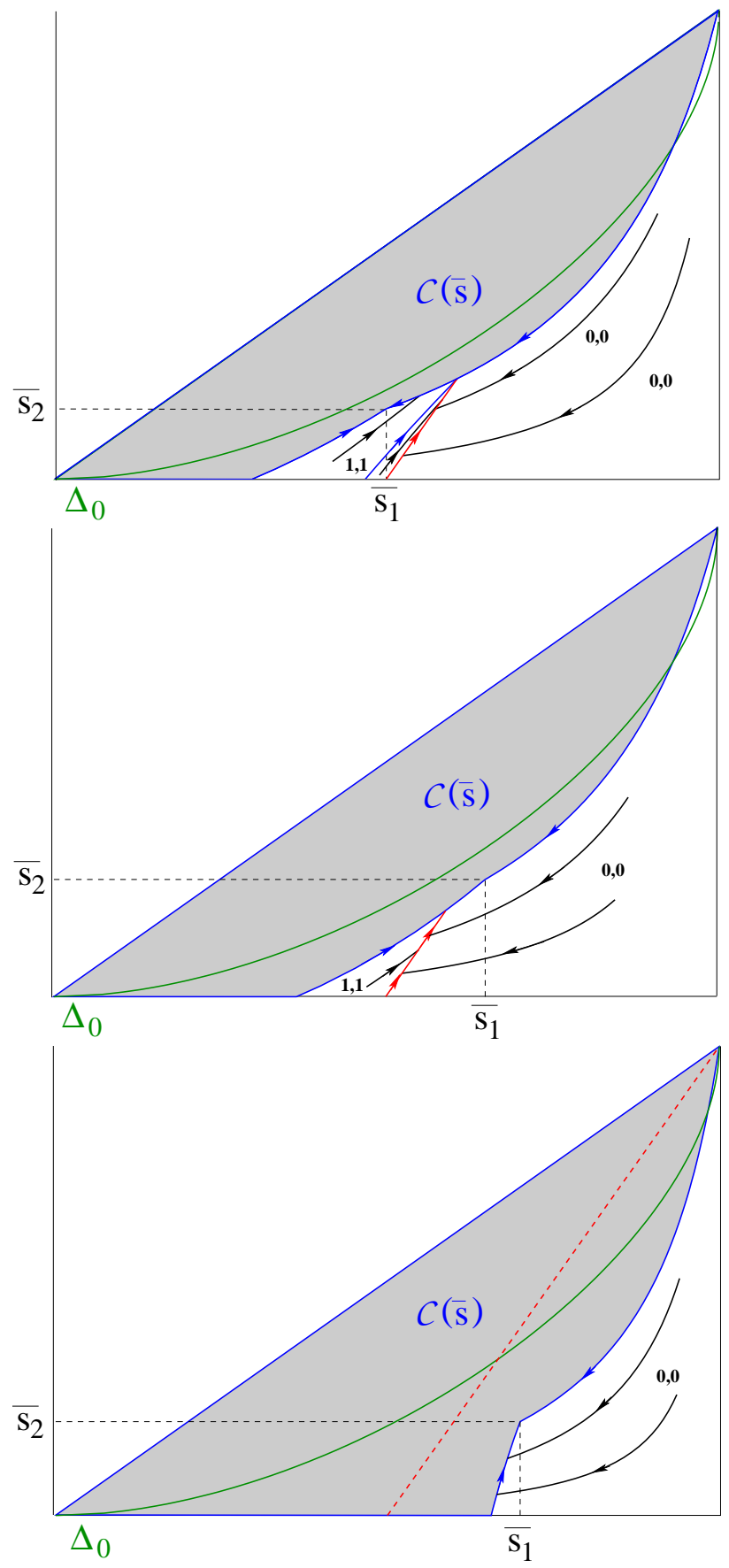

FiguRE 7. Examples of optimal trajectories (in black) for different targets $\bar{s}$ in the case $s^{\star} \in \mathcal{C}(\bar{s})$ with $\mu(s)=s$. First picture: target in $\Delta_{S A}^{-}$. Second picture: target in $\Delta_{S A}^{+}$. Third picture: the singular arc satisfies $\Delta_{S A} \subset \mathcal{C}(\bar{s})$ and the singular strategy reduces to $u=0$ until reaching $\partial \mathcal{C}(\bar{s})$

target is reachable or not, and the nature of the optimal feedback depending on the position of the initial condition with respect to these curves. 


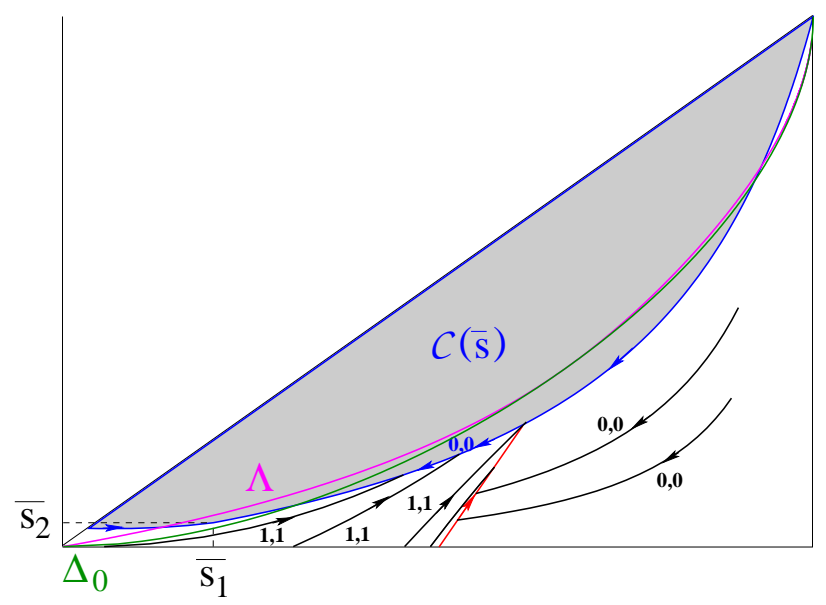

FIGURE 8. In purple: the semi-permeable curve $\Lambda$ with $\mu(s)=s$. In green: the curve $\Delta_{0}$. In blue: the boundary of $\mathcal{C}(\bar{s})$. In red: the singular arc $\Delta_{S A}$. In black: optimal trajectories for different initial state outside of $\mathcal{C}(\bar{s})$.

Acknowledgments. We thank Professor Denis Dochain (UCL, Louvain, Belgium) for indicating this problem in the framework of the European project CAFE, and José Fernandez, a former PhD student, that has started to work on this problem. The authors would like to thank E. Trélat for fruitful discussion on the subject. The first author thanks Inria for providing him a one year research opportunity at INRA-Inria project MODEMIC. The third author thanks the Inria Chile, INRA-Inria project MODEMIC and CONICYT through project REDES130067 (Center for Mathematical Modeling, Universidad de Chile) for providing him a postdoctoral fellowship. The authors express their thanks to the anonymous referees for their comments.

\section{REFERENCES}

[1] D. Ami, P. Cartigny And A. Rapaport, Can marine protected areas enhance both economic and biological situations?, Comptes Rendus Biologies, 328, 4 (2005), pp. 357-366.

[2] W. Bakker, H. Beeftink, C. De Gooijer and J. Tramper, Bioreactors in series: An overview of design procedures and practical applications, Enzyme and Microbial Technology, 18 (1996), pp. 202-219.

[3] M. Bardi, I. Capuzzo-Dolcetta, Optimal Control and Visosity Solutions of Hamilton-JacobiBellman Equations, Birkhauser, 1997.

[4] T. Bayen, F. Mairet, P. Gajardo, Optimal synthesis for the minimum time control problems of fed-batch bioprocesses for growth functions with two maxima, Journal of Optim. Theory and Applications, 158, 2 (2013), pp. 521-553.

[5] B. Bonnard And M. Chyba, Singular Trajectories and their role in Control Theory, Springer, SMAI, 40, 2002.

[6] U. Boscain and B. Piccoli, Optimal Syntheses for Control Systems on 2-D Manifolds, Springer SMAI, 43, 2004.

[7] P. Cadarliaguet, On the regularity of semipermeable surfaces in control theory with application to the optimal exit-time problem (part I), SIAM J. Control Optim, 35, 5 (1997), pp. 1638-1652.

[8] P. Cadarliaguet, On the regularity of semipermeable surfaces in control theory with application to the optimal exit-time problem (part II), SIAM J. Control Optim, 35, 5 (1997), pp. 1653-1671.

[9] C. Clark, Mathematical Bioeconomics: The Optimal Management of Renewable Resources, John Wiley \& Sons (2nd ed.), 1990.

[10] I. Cacciari, S. GRego And E. Di Mattia, Eco-physiological characterization of soil bacterial 
populations in different states of growth, Microb. Ecol. 43, 1 (2002), pp. 34-43.

[11] C. Camarasa, T. Clement, M. Perez, J.R. Mouret and J.M. Sablayrolles, Use of a continuous multistage bioreactor to mimic winemaking fermentation, International Journal of Food Microbiology, 150, 1 (2011), pp. 42-49.

[12] D. Dochain And A. Rapaport, Minimal time control of fed-batch processes for growth functions with several maxima, IEEE Transactions on Automatic Control, 56, 11 (2011), pp. 2671-2676.

[13] A. Dramé, J. Harmand, C. Lobry and A. Rapaport, Multiple steady state profiles in interconnected biological systems, Mathematical and Computer Modelling of Dynamical Systems, 12 (2006), pp. 379-393.

[14] B. Dubey, P. Chandra and P. Sinha, A model for fishery resource with reserve area, Nonlinear Analysis: Real World Applications 4 (2003), pp. 625-637.

[15] B. Bonnard, J.-B. Caillau, E. Trélat, Second order optimality conditions in the smooth case and applications in optimal control, ESAIM Control Optim. Calc. 13, 2 (2007), pp. $207-236$.

[16] D. Dochain and P. Vanrolleghem, Dynamical Modelling and Estimation in Wastewater treatment Processes, IWA Publishing, U.K., 2001.

[17] H. El-Owaidy and O. El-Leithy, Theoretical studies on extinction in the gradostat, Mathematical Biosciences, 101, 1 (1990), pp. 1-26.

[18] P. Gajardo, H. RAmirez, A. RAPaport, Minimal time sequential batch reactors with bounded and impulse controls for one or more species, SIAM J. Control Optim., 47, 6 (2008), pp. $2827-2856$.

[19] F. GÉrard, I. Haidar And A. RApaport, Effects of spatial structure and diffusion on the performances of the chemostat, Mathematical Biosciences and Engineering, 8, 4 (2011), pp. 953-971.

[20] R. Hannesson, Marine Reserves: What would they Accomplish?, Marine Resource economics, 13 (1998), pp. 159-170.

[21] J. Harmand, A. RAPAPort And A. Trofino, Optimal design of two interconnected bioreactors-some new results, American Institute of Chemical Engineering Journal, 49 (1999), pp. 1433-1450.

[22] Y. Higashi, N. Ytow, H. Saida And H. Seki, In situ gradostat for the study of natural phytoplankton community with an experimental nutrient gradient Environmental Pollution, 99 (1998), pp. 395-404.

[23] G. Hill and C. Robinson, Minimum tank volumes for CFST bioreactors in series, The Canadian Journal of Chemical Engineering, 67 (1989), pp. 818-824.

[24] E.B. Lee, L. Markus, Foundations of optimal control theory, John Wiley \& Sons, Inc., New York, 1967.

[25] U. Ledzewicz and H. Schattler, Geometric Optimal Control, Springer, New-York, 2012.

[26] R. Lovitt and J. Wimpenny, The gradostat: A bidirectional compound chemostat and its applications in microbial research, Journal of General Microbiology, 127 (1981), pp. 261268.

[27] A. Miele, Application of Green's Theorem to the extremization of linear integrals, Symp. on Vehicle Systems Optimization, Garden City, L. I. New York (1961),pp. 26-35.

[28] K. Mischaikow, H.L. Smith, H. Thieme Asymptotically autonomous semiflows: chain recurrence and Liapunov functions, Trans. Amer. Math. Soc., 347 (1995), pp. 1669-1685.

[29] J. A. Moreno, Optimal time control of bioreactors for the wastewater treatment, Optim. Control Appl. Meth., 20 (1999), pp. 145-164.

[30] M. Nelson And H. Sidhu, Evaluating the performance of a cascade of two bioreactors, Chemical Engineering Science, 61 (2006), pp. 3159-3166.

[31] M. Quincampoix, Differential inclusions and target problems, SIAM J. Control Optim., 30, 2 (1992), pp. 324-335.

[32] J.A. Sanchirico And J.E. Wilen, Bioeconomics of Spacial Exploitation in a Patchy Environment, Journal of Environmental Economics and Management, 37 (1999), pp. 129-15.

[33] H.L. Smith And P. Waltman, The gradostat: a model of competition along a nutrient gradient, J. Microb. Ecol., 22 (1991), pp. 207-226.

[34] H.L. Smith And P. Waltman, The theory of the chemostat, Dynamics of microbial competition, Cambridge University Press, 1995.

[35] B. TANG, Mathematical investigations of growth of microorganisms in the gradostat, J. Math. Biol., 23 (1986), pp. 319-339.

[36] H. VeldCAmp, Ecological studies with the chemostat, Advances in Microbial Ecology, 1 (1977), pp. 59-95. 